\title{
An Efficient Linear Scheme to Approximate Parabolic Free Boundary Problems: Error Estimates and Implementation*
}

\author{
By Ricardo H. Nochetto and Claudio Verdi
}

\begin{abstract}
This paper deals with a fully discrete scheme to approximate multidimensional singular parabolic problems; two-phase Stefan problems and porous medium equations are included. The algorithm consists of approximating at each time step a linear elliptic partial differential equation by piecewise linear finite elements and then making an element-by-element algebraic correction to account for the nonlinearity. Several energy error estimates are derived for the physical unknowns; a sharp rate of convergence of $O\left(h^{1 / 2}\right)$ is our main result. The crucial point in implementing the scheme is the efficient resolution of linear systems involved. This topic is discussed, and the results of several numerical experiments are shown.
\end{abstract}

1. Introduction. The aim of this paper is to analyze from both a theoretical and computational viewpoint the performance of a linear scheme to approximate the following multidimensional parabolic problem:

$$
\begin{aligned}
\frac{\partial u}{\partial t}-\Delta \beta(u) & =f(\beta(u)) & & \text { in } Q:=\Omega \times(0, T), \\
\beta(u) & =0 & & \text { on } \partial \Omega \times(0, T), \\
u(0) & =u_{0} . & &
\end{aligned}
$$

Here, $\beta$ stands for a nondecreasing Lipschitz continuous function defined on $\mathbf{R}$ and $\Omega$ for a polyhedral and convex domain in $\mathbf{R}^{d}(d \geq 1)$. The geometrical constraints upon $\Omega$, as well as the type of boundary condition considered, were chosen only for the sake of simplicity. It is well known that formulation (1.1) is so general as to include two-phase Stefan problems and porous medium equations.

The usual technique to approximate (1.1) (with or without regularization of $\beta$ ) amounts to discretizing a nonlinear elliptic partial differential equation at each time step. Both, theoretical and numerical results, are now well known for these schemes [10], [23], [16], [17], [18], [8], [20], [25] which behave rather well in approximating not only solutions, but also interfaces [19], [20]. However, when dealing with nonlinear problems, one usually tries to linearize them so as to take advantage of efficient linear solvers [7], [15]. The success of such a procedure relies on the smoothness of the solutions $u$ and $\theta:=\beta(u)$. Consequently, it is not a priori obvious that standard

Received April 2, 1987.

1980 Mathematics Subject Classification (1985 Revision). Primary 65N15, 65N30; Secondary $35 \mathrm{~K} 55,35 \mathrm{~K} 65,35 \mathrm{R} 35$.

* This research was supported in part by the Institute for Mathematics and its Applications with funds provided by the N.S.F. and by M.P.I.-Fondi per la ricerca scientifica-and Istituto di Analisi Numerica del C.N.R. di Pavia (Italy). 
techniques for mildly nonlinear parabolic equations apply in this context, because (1.1) is just a low-regularity problem. Moreover, high-order accuracy schemes in both space and time may be useless here, again because of the lack of regularity. So the question is how to linearize (1.1) properly.

Our present purpose is to discuss the stability and approximating properties of a fully discrete linear scheme associated with the following discrete-time scheme (which is a nonlinear Chernoff formula):

$$
\begin{aligned}
& U^{0}:=u_{0}, \\
& \Theta^{n}-\frac{\tau}{\mu} \Delta \Theta^{n}=\beta\left(U^{n-1}\right)+\frac{\tau}{\mu} f\left(\beta\left(U^{n-1}\right)\right), \\
& U^{n}:=U^{n-1}+\mu\left[\Theta^{n}-\beta\left(U^{n-1}\right)\right], \quad 1 \leq n \leq N:=\frac{T}{\tau} .
\end{aligned}
$$

Here, $\tau>0$ is the time step and $\mu>0$ is the relaxation parameter which satisfies the stability constraint $\mu \leq L_{\beta}^{-1}\left(L_{\beta}=\right.$ Lipschitz constant of $\left.\beta\right)$. This discretetime algorithm was studied in an abstract and general setting by Brezis and Pazy [4] and was first used in numerical analysis by Berger, Brezis and Rogers [2] (see also Verdi [24] and Magenes and Verdi [14]), who showed its convergence. Sharp energy error estimates for both singular and mildly nonlinear parabolic problems were recently proved by Magenes, Nochetto and Verdi [13]. The error analysis has been extended by Magenes [12] to other algorithms suggested by nonlinear semigroup theory. The nonlinear Chernoff formula (1.2) can be regarded as the discrete-time phase relaxation scheme introduced by Visintin [27] and Verdi and Visintin [26]. This point of view was essential in [13], because it exhibits the variational structure of (1.2). Moreover, it allowed the use of variational techniques, first applied by Nochetto [16], [17], [18] and Nochetto and Verdi [20] for analyzing singular parabolic problems and dealing with minimal regularity properties (say, $u_{0} \in L^{2}(\Omega)$ ), respectively. We remark, in addition, that (1.2) is in the same spirit as the Laplace-modified forward Galerkin method of Douglas and Dupont [7] for nondegenerate parabolic equations.

The algorithm (1.2) actually gives rise to an effective numerical scheme after discretizing in space; namely, the variable $\Theta^{n}$ is approximated by continuous piecewise linear finite elements and the variable $U^{n}$ by piecewise constants. The primary aim of this paper is to show that the resulting scheme is stable and preserves the approximation properties of (1.2). The tools in deriving the rates of convergence are essentially those in [13], [26]. The crucial step in implementing the fully discrete scheme is the efficient resolution of the linear elliptic partial differential equation in (1.2). We used an incomplete Cholesky factorization for preconditioning the resulting matrix, coupled with a conjugate gradient method [1], [15]. We chose this iterative technique because we had in mind an automatic decomposition of a general domain $\Omega$, rather than a particular geometry. Therefore, we cannot take advantage of geometrical properties of $\Omega$ which allow direct methods to be competitive. The numerical experiments confirm the ability of our scheme to approximate solutions. The observed orders of convergence agree with the theoretical ones. The approximation of the interfaces is not as good as for the usual nonlinear scheme [20]; this is due to artificial diffusion added by the linear partial differential equation in $(1.2)$. 
The outline of the paper is as follows. Section 2 is devoted to stating the assumptions and the continuous and fully discrete problems. The stability of the discrete scheme in energy and maximum norms is proved in Section 3. Several energy error estimates for both physical unknowns $u$ and $\theta=\beta(u)$ are derived in Section 4. Finally, the implementation of the method, as well as the results of several numerical experiments, are discussed in Section 5.

2. Formulation of the Problem. In this section we shall establish the hypotheses on the data and state the continuous and the fully discrete problems.

2.1. Basic Assumptions, Notation and Finite Element Properties. We make the following assumptions:

$\Omega \subset \mathbf{R}^{d}(d \geq 1)$ is a polyhedral and convex domain. Set $Q:=$ $\Omega \times(0, T)$, where $0<T<+\infty$ is fixed.

$\beta: \mathbf{R} \rightarrow \mathbf{R}, \beta(0)=0$, is a nondecreasing and Lipschitz continuous function; more precisely,

$\left(\mathrm{H}_{\beta}\right)_{1}$

$$
0 \leq \beta^{\prime}(s) \leq L_{\beta}<+\infty \text { for a.e. } s \in \mathbf{R}
$$

moreover, $\beta$ grows at least linearly at infinity,

$$
\exists C_{1}, C_{2}>0: \quad \forall s \in \mathbf{R},|s| \leq C_{1}+C_{2}|\beta(s)| .
$$

$$
f: \mathbf{R} \rightarrow \mathbf{R} \text { is a uniformly Lipschitz continuous function, }
$$

$$
\left|f\left(s_{1}\right)-f\left(s_{2}\right)\right| \leq L_{f}\left|s_{1}-s_{2}\right| \quad \forall s_{1}, s_{2} \in \mathbf{R} .
$$

The geometrical constraints on $\Omega$, as well as the fact that $\beta$ and $f$ are independent of the space and time variables $(x, t)$, were assumed only for the sake of simplicity. Consequently, a more general treatment is still possible (see Remark 11). The basic regularity required on the initial datum is

$$
\left(\mathrm{H}_{u_{0}}\right) \quad u_{0} \in L^{2}(\Omega) \text {. }
$$

This assumption will be strengthened later on in order to prove some of our results.

Let $\left\{\mathbf{S}_{h}\right\}_{h}$ be a family of decompositions $\mathbf{S}_{h}=\left\{S_{k}\right\}_{k=1}^{K}$ of $\Omega$ into closed $d$ simplices, so that $\bar{\Omega}=\bigcup_{k=1}^{K} S_{k}$; as usual, $h$ stands for the mesh size. We assume that

$\left(\mathbf{H}_{\mathbf{S}_{h}}\right) \quad$ the family $\left\{\mathbf{S}_{h}\right\}_{h}$ is regular [5, p. 132].

Since quasi-uniformity is not required, local refinements are allowed. A further property we need only in Lemma 2 is the acuteness of $\left\{\mathbf{S}_{h}\right\}_{h}$; this guarantees the discrete maximum principle to hold [6]. Let us now define the discrete spaces we shall work with:

$$
\begin{aligned}
& V_{h}^{1}:=\left\{\chi \in C^{0}(\bar{\Omega}):\left.\chi\right|_{S_{k}} \text { is linear } \forall k=1, \ldots, K, \chi=0 \text { on } \partial \Omega\right\}, \\
& V_{h}^{0}:=\left\{\psi:\left.\psi\right|_{S_{k}} \text { is constant } \forall k=1, \ldots, K\right\} .
\end{aligned}
$$

We denote by $\langle\cdot, \cdot\rangle$ both the inner product in $L^{2}(\Omega)$ and the duality pairing between $H^{-1}(\Omega)$ and $H_{0}^{1}(\Omega)$. The corresponding discrete inner product is defined by

$$
\langle\chi, \phi\rangle_{h}:=\sum_{k=1}^{K} \int_{S_{k}} \Pi_{h}(\chi \phi) d x
$$


for any piecewise uniformly continuous functions $\chi$ and $\phi$, where $\Pi_{h}$ stands for the local linear interpolation operator. Notice that the integral in (2.1) can be evaluated easily by means of the vertex quadrature rule which is exact for piecewise linear functions [5, p. 182]. It is well known that $\langle\cdot, \cdot\rangle_{h}$ is an inner product in $V_{h}^{1}$ which satisfies [22, p. 260]

$$
\|\chi\|_{L^{2}(\Omega)}^{2} \leq\langle\chi, \chi\rangle_{h} \leq C\|\chi\|_{L^{2}(\Omega)}^{2} \quad \forall \chi \in V_{h}^{1}
$$

where $C \geq 1$ is a constant independent of $h$. The following well-known error bound takes into account the effect of numerical integration:

$$
\left|\langle\chi, \phi\rangle-\langle\chi, \phi\rangle_{h}\right| \leq C h^{2}\|\nabla \chi\|_{L^{2}(\Omega)}\|\nabla \phi\|_{L^{2}(\Omega)} \quad \forall \chi, \phi \in V_{h}^{1} .
$$

We now introduce the discrete $H^{1}$-projection operator $P_{h}^{1}$; more precisely, for any $z \in H_{0}^{1}(\Omega)$, let $P_{h}^{1} z \in V_{h}^{1}$ be defined by

$$
\left\langle\nabla P_{h}^{1} z, \nabla \chi\right\rangle=\langle\nabla z, \nabla \chi\rangle \quad \forall \chi \in V_{h}^{1} .
$$

Since $\left\{\mathbf{S}_{h}\right\}_{h}$ and the Green operator associated with the inner product in $H_{0}^{1}(\Omega)$ are regular, the following approximation property holds for any $z \in H_{0}^{1}(\Omega)[5, \mathrm{p}$. 138]:

$$
\left\|z-P_{h}^{1} z\right\|_{H^{r}(\Omega)} \leq C h\|z\|_{H^{r+1}(\Omega)}, \quad r=0,1 .
$$

We also introduce the $L^{2}$-projection operator $P_{h}^{0}$ onto $V_{h}^{0}$ which, for any $z \in L^{2}(\Omega)$, is defined by

$$
\left\langle P_{h}^{0} z, \psi\right\rangle=\langle z, \psi\rangle \quad \forall \psi \in V_{h}^{0}
$$

and satisfies

$$
\left\|z-P_{h}^{0} z\right\|_{H^{-s}(\Omega)} \leq C h^{r+s}\|z\|_{H^{r}(\Omega)}, \quad 0 \leq s, r \leq 1 .
$$

We conclude with some notation concerning the time discretization. Let $\tau:=T / N$ be the time step $(N$ a positive integer $)$ and set $t^{n}:=n \tau, I^{n}:=\left(t^{n-1}, t^{n}\right]$ for $1 \leq n \leq N$. W'e also set

$$
z^{n}:=z\left(\cdot, t^{n}\right), \quad \bar{z}^{n}:=\frac{1}{\tau} \int_{I^{n}} z(\cdot, t) d t \quad\left(\bar{z}^{0}:=z^{0}\right)
$$

for any continuous (resp. integrable) function in time defined in $Q$, and

$$
\partial z^{n}:=\frac{z^{n}-z^{n-1}}{\tau}, \quad 1 \leq n \leq N
$$

for any given family $\left\{z^{n}\right\}_{n=0}^{N}$.

2.2. The Continuous Problem: Regularity. We now state the variational formulation of problem (1.1) we shall work with.

Problem (P): Find $\{u, \theta\}$ such that

$$
\begin{gathered}
u \in L^{\infty}\left(0, T ; L^{2}(\Omega)\right) \cap H^{1}\left(0, T ; H^{-1}(\Omega)\right), \quad \theta \in L^{2}\left(0, T ; H_{0}^{1}(\Omega)\right), \\
\theta(x, t)=\beta(u(x, t)) \quad \text { for a.e. }(x, t) \in Q, \\
u(\cdot, 0)=u_{0}
\end{gathered}
$$

and for a.e. $t \in(0, T)$ and for all $\phi \in H_{0}^{1}(\Omega)$ the following equation holds,

$$
\left\langle\frac{\partial u}{\partial t}, \phi\right\rangle+\langle\nabla \theta, \nabla \phi\rangle=\langle f(\theta), \phi\rangle \text {. }
$$


Existence and uniqueness are well known for (P) (see, e.g., [9], [11] and the references given therein).

Next, we recall the further regularity results we shall use in the sequel. Letting assumptions $\left(\mathrm{H}_{\Omega}\right),\left(\mathrm{H}_{\beta}\right)_{1},\left(\mathrm{H}_{f}\right)$ and $\left(\mathrm{H}_{u_{0}}\right)$ hold, we have that

(R1) if $u_{0} \in L^{\infty}(\Omega)$, then $u, \theta \in L^{\infty}(Q)$;

(R2) if $\Delta \beta\left(u_{0}\right) \in L^{1}(\Omega)$, then $\partial u / \partial t \in L^{\infty}(0, T ; M(\Omega))$, where $M(\Omega)$ denotes the set of finite regular Baire measures [10], [23]; [9], [11].

Remark 1. We stress that the assumption on the initial datum in (R2) may be weakened somewhat by taking $\Delta \beta\left(u_{0}\right)=l+\mu$, where $l \in L^{1}(\Omega)$ and $\mu \in M(\Omega)$, provided that the set $S_{0}:=\partial\left\{x \in \Omega: \beta\left(u_{0}(x)\right)=0\right\} \cap \Omega$ is sufficiently smooth and supp $\mu \subset S_{0}$. The proof proceeds as in [11] after a suitable regularization.

2.3. The Fully Discrete Nonlinear Chernoff Formula. Finally, let us state the fully discrete algorithm precisely. Let $0<\mu \leq L_{\beta}^{-1}$ be a fixed number (the so-called relaxation parameter).

Problem $\left(\mathrm{P}_{h, \tau}\right):$ For any $1 \leq n \leq N$, find $\left\{U^{n}, \Theta^{n}\right\}$ such that $U^{n} \in V_{h}^{0}, \Theta^{n} \in V_{h}^{1}$ and, setting

$$
U^{0}:=P_{h}^{0} u_{0}
$$

we have

$$
\left\langle\Theta^{n}, \chi\right\rangle_{h}+\frac{\tau}{\mu}\left\langle\nabla \Theta^{n}, \nabla \chi\right\rangle=\left\langle\beta\left(U^{n-1}\right)+\frac{\tau}{\mu} f\left(\beta\left(U^{n-1}\right)\right), \chi\right\rangle
$$

for all $\chi \in V_{h}^{1}$, and

$$
U^{n}=U^{n-1}+\mu\left[P_{h}^{0} \Theta^{n}-\beta\left(U^{n-1}\right)\right]
$$

Since the matrix of the linear system (2.11) is symmetric and positive definite, the solution of $\left(\mathrm{P}_{h, \tau}\right)$ exists and is unique (see also Section 5). Moreover, since both $U^{n}$ and $P_{h}^{0} \Theta^{n}$ are piecewise constants, Eq. (2.12) may be regarded as an elementby-element algebraic correction which takes into account the intrinsic nonlinearity.

Remark 2. For computational convenience we would like to take the piecewise constant interpolant of $u_{0}$ as initial datum $U^{0}$, rather than $P_{h}^{0} U_{0}$. This is simply impossible, in general, owing to the lack of regularity of $u_{0}$, but it is still possible in some cases of relevant interest for both Stefan problems and porous medium equations. Namely, assume that the phases are initially separated by smooth surfaces, say Hölder continuous, and also that $u_{0}$ is regular in each phase, say $u_{0} \in C^{0,1 / 2}$. We then define $U^{0}$ to be either the value of $u_{0}$ at the barycenter $y_{k}$ of the simplex $S_{k}$, whenever $y_{k}$ belongs to the interior of the phases, or any value in the range of $u_{0}$ restricted to $S_{k}$, provided $y_{k}$ lies on the initial interfaces. It is easily seen that

$$
\left\|u_{0}-U^{0}\right\|_{L^{2}(\Omega)}=O\left(h^{1 / 2}\right)
$$

this error estimate suffices for later purposes.

3. Stability of the Discrete Scheme. We start by combining the equations (2.11) and (2.12) and rewriting the discrete problem as follows:

$$
\left\langle\partial U^{n}, \chi\right\rangle+\left\langle\nabla \Theta^{n}, \nabla \chi\right\rangle=\frac{\mu}{\tau}\left\langle\left[P_{h}^{0}-I\right] \Theta^{n}, \chi\right\rangle_{h}+\left\langle f\left(\beta\left(U^{n-1}\right)\right), \chi\right\rangle \quad \forall \chi \in V_{h}^{1}
$$


Since the relaxation parameter $\mu$ is chosen so that $0<\mu \leq L_{\beta}^{-1}$, the following property holds:

$$
\alpha:=I-\mu \beta \text { satisfies } 0 \leq \alpha^{\prime}(s) \leq 1 \text { for a.e. } s \in \mathbf{R} .
$$

The proof of the a priori estimates proceeds along the same lines as that for the semidiscrete approximation analyzed in [13]. However, for the sake of completeness, we present here a detailed proof.

3.1. Stability in Energy Norms. Given an absolutely continuous function $\lambda: \mathbf{R} \rightarrow$ $\mathbf{R}$ so that $\lambda(0)=0$ and $0 \leq \lambda^{\prime} \leq \Lambda<\infty, \Phi_{\lambda}$ stands for the convex function defined by

$$
\Phi_{\lambda}(s):=\int_{0}^{s} \lambda(z) d z \quad \text { for } s \in \mathbf{R} .
$$

$\Phi_{\lambda}$ has the properties

$$
\frac{1}{2 \Lambda} \lambda^{2}(s) \leq \Phi_{\lambda}(s) \leq \frac{\Lambda}{2} s^{2} \quad \text { for } s \in \mathbf{R},
$$

which are easily proved. The following elementary relations will be used in the sequel:

$$
\begin{gathered}
2 a b \leq \eta a^{2}+b^{2} / \eta \quad \text { for } a, b \in \mathbf{R}, \eta>0 ; \\
2 a[a-b]=a^{2}-b^{2}+[a-b]^{2} \quad \text { for } a, b \in \mathbf{R} .
\end{gathered}
$$

LEMMA 1. Assume that $\left(\mathrm{H}_{\Omega}\right),\left(\mathrm{H}_{\beta}\right)_{1},\left(\mathrm{H}_{f}\right),\left(\mathrm{H}_{u_{0}}\right)$ and $\left(\mathrm{H}_{\mathbf{S}_{h}}\right)$ hold. Then there exists a constant $C>0$ independent of the discretization parameters such that

$$
\max _{1 \leq n \leq N}\left\|\beta\left(U^{n}\right)\right\|_{L^{2}(\Omega)}+\sum_{n=1}^{N}\left\|U^{n}-U^{n-1}\right\|_{L^{2}(\Omega)}^{2}+\sum_{n=1}^{N} \tau\left\|\Theta^{n}\right\|_{H^{1}(\Omega)}^{2} \leq C .
$$

The norm in the middle may be viewed as a discrete $H^{1 / 2}\left(0, T ; L^{2}(\Omega)\right)$ norm. The constant $C$ in (3.6) is proportional to $\mu^{-1} \exp \left(C^{*} L_{f} \mu^{-1} T\right)$ as $\mu \downarrow 0$, where $C^{*}>0$ is a universal constant.

Proof of Lemma 1. The function $\Theta^{n}$ is an admissible test function in (3.1), because $\Theta^{n} \in V_{h}^{1}$. So let us take $\chi=\tau \Theta^{n}$ and sum (3.1) over $n$ from 1 to $m \leq N$. We proceed to estimate each resulting term. First note that

$$
\begin{aligned}
P_{h}^{0} \Theta^{n} & =\frac{1}{\mu}\left[U^{n}-U^{n-1}\right]+\beta\left(U^{n-1}\right) \\
& =\frac{1}{2} \beta\left(U^{n}\right)-\frac{1}{2 \mu} \alpha\left(U^{n-1}\right)+\frac{1}{2 \mu} U^{n}+\frac{1}{2 \mu}\left[\alpha\left(U^{n}\right)-\alpha\left(U^{n-1}\right)\right] .
\end{aligned}
$$

Next, using the definition of the $L^{2}$-projection $P_{h}^{0}$, the convexity of $\Phi_{\beta}$ and $\Phi_{\alpha}$ and the identity (3.5), we can bound the first term in (3.1) from below; namely,

$$
\begin{aligned}
2 \sum_{n=1}^{m}\left\langle U^{n}-U^{n-1}, \Theta^{n}\right\rangle=2 \sum_{n=1}^{m}\left\langle U^{n}-U^{n-1}, P_{h}^{0} \Theta^{n}\right\rangle \\
\quad \geq \int_{\Omega} \sum_{n=1}^{m}\left\{\left[\Phi_{\beta}\left(U^{n}\right)-\Phi_{\beta}\left(U^{n-1}\right)\right]+\frac{1}{\mu}\left[\Phi_{\alpha}\left(U^{n-1}\right)-\Phi_{\alpha}\left(U^{n}\right)\right]\right\} d x \\
\quad+\frac{1}{2 \mu}\left[\left\|U^{m}\right\|_{L^{2}(\Omega)}^{2}-\left\|U^{0}\right\|_{L^{2}(\Omega)}^{2}+\sum_{n=1}^{m}\left\|U^{n}-U^{n-1}\right\|_{L^{2}(\Omega)}^{2}\right]
\end{aligned}
$$


Here, we have also used that $\alpha$ is increasing, in order to eliminate the contribution of the last term in (3.7). The terms involving the functions $\Phi_{\beta}$ and $\Phi_{\alpha}$ can be further bounded by means of (3.2) and (3.3) as follows:

$$
\begin{gathered}
\int_{\Omega} \sum_{n=1}^{m}\left[\Phi_{\beta}\left(U^{n}\right)-\Phi_{\beta}\left(U^{n-1}\right)\right]=\int_{\Omega}\left[\Phi_{\beta}\left(U^{m}\right)-\Phi_{\beta}\left(U^{0}\right)\right] \\
\geq \frac{1}{2 L_{\beta}}\left\|\beta\left(U^{m}\right)\right\|_{L^{2}(\Omega)}^{2}-\frac{L_{\beta}}{2}\left\|U^{0}\right\|_{L^{2}(\Omega)}^{2}
\end{gathered}
$$

and

$$
\begin{gathered}
\frac{1}{\mu} \int_{\Omega} \sum_{n=1}^{m}\left[\Phi_{\alpha}\left(U^{n-1}\right)-\Phi_{\alpha}\left(U^{n}\right)\right]=\frac{1}{\mu} \int_{\Omega}\left[\Phi_{\alpha}\left(U^{0}\right)-\Phi_{\alpha}\left(U^{m}\right)\right] \\
\geq \frac{1}{2 \mu}\left\|\alpha\left(U^{0}\right)\right\|_{L^{2}(\Omega)}^{2}-\frac{1}{2 \mu}\left\|U^{m}\right\|_{L^{2}(\Omega)}^{2} .
\end{gathered}
$$

Inserting these estimates into the previous expression and using assumption $\left(\mathrm{H}_{u_{0}}\right)$ leads to

$$
\sum_{n=1}^{m}\left\langle U^{n}-U^{n-1}, \Theta^{n}\right\rangle \geq-C+C\left\|\beta\left(U^{m}\right)\right\|_{L^{2}(\Omega)}^{2}+\frac{1}{4 \mu} \sum_{n=1}^{m}\left\|U^{n}-U^{n-1}\right\|_{L^{2}(\Omega)}^{2} .
$$

The next term on the left-hand side of (3.1) provides the $H^{1}$-estimate, because Poincaré's inequality holds. The first term on the right is nonpositive; indeed, the orthogonality property of the $L^{2}$-projection $P_{h}^{0}$ implies

$$
\mu \sum_{n=1}^{m}\left\langle\left[P_{h}^{0}-I\right] \Theta^{n}, \Theta^{n}\right\rangle_{h}=\mu \sum_{n=1}^{m}\left\langle\left[P_{h}^{0}-I\right] \Theta^{n},\left[I-P_{h}^{0}\right] \Theta^{n}\right\rangle_{h} \leq 0 .
$$

We now analyze the contribution due to the source term. In view of the definition of $P_{h}^{0}$ and assumption $\left(\mathrm{H}_{f}\right)$, combined with the first equality in (3.7) and (3.4), we get

$$
\begin{aligned}
& \left|\sum_{n=1}^{m} \tau\left\langle f\left(\beta\left(U^{n-1}\right)\right), \Theta^{n}\right\rangle\right|=\left|\sum_{n=1}^{m} \tau\left\langle f\left(\beta\left(U^{n-1}\right)\right), P_{h}^{0} \Theta^{n}\right\rangle\right| \\
& \quad \leq C+C \sum_{n=1}^{m} \tau\left\|\beta\left(U^{n-1}\right)\right\|_{L^{2}(\Omega)}^{2}+\frac{1}{8 \mu} \sum_{n=1}^{m}\left\|U^{n}-U^{n-1}\right\|_{L^{2}(\Omega)}^{2} .
\end{aligned}
$$

Since the last term may be absorbed into the left-hand side of (3.1), the assertion (3.6) follows as a consequence of the discrete Gronwall inequality.

Remark 3. From (3.6) and the first equality in (3.7) it is easily seen that

$$
\max _{1 \leq n \leq N}\left\|P_{h}^{0} \Theta^{n}\right\|_{L^{2}(\Omega)} \leq C .
$$

Remark 4. If $\beta$ grows at least linearly at infinity, as stated in $\left(\mathrm{H}_{\beta}\right)_{2}$, the first term in (3.6) provides the a priori bound

$$
\max _{1 \leq n \leq N}\left\|U^{n}\right\|_{L^{2}(\Omega)} \leq C .
$$

3.2. Stability in Maximum Norm. The present goal is to prove an a priori estimate in $L^{\infty}(\Omega)$. To this end, we need a further assumption on the triangulation, 
namely that $\mathbf{S}_{h}$ is of acute type. In other words,

The projection of the vertices of any $d$-simplex $S \in \mathbf{S}_{h}$ onto the hyperplane containing the opposite face lies in the closure of this face.

Remark 5. In 2-D, the above constraint means that internal angles of any triangle of the decomposition do not exceed $\pi / 2$.

We then have the following well-known discrete maximum principle for the Laplace operator [6]:

Let $\chi \in V_{h}^{1}$ attain its maximum at the internal node $x_{j}$ and let $\phi^{j} \in V_{h}^{1}$ be the corresponding basis function. Then

$$
\int_{\Omega} \nabla \chi \cdot \nabla \phi^{j} \geq 0
$$

Now we are ready to prove the desired $L^{\infty}$-estimate.

LEMMA 2. Assume that $\left(\mathrm{H}_{\Omega}\right),\left(\mathrm{H}_{\beta}\right)_{1},\left(\mathrm{H}_{f}\right),\left(\mathrm{H}_{\mathbf{S}_{h}}\right)$ and $(3.10)$ hold and in addition that

$$
u_{0} \in L^{\infty}(\Omega) .
$$

Then there exists a constant $C>0$ independent of the approximation parameters such that

$$
\max _{1 \leq n \leq N}\left\|U^{n}\right\|_{L^{\infty}(\Omega)} \leq C .
$$

Proof. What we actually want to prove is the following estimate,

$$
\left\|U^{n}\right\|_{L^{\infty}(\Omega)} \leq C_{n}:=C_{0} e^{n \tau L_{f} L_{\beta}}+\frac{f_{0}}{L_{f} L_{\beta}}\left[e^{n \tau L_{f} L_{\beta}}-1\right], \quad 1 \leq n \leq N,
$$

which obviously implies the assertion (3.13). Here, $C_{0}$ stands for a positive constant such that $-C_{0} \leq U^{0}=P_{h}^{0} u_{0} \leq C_{0}$ in $\Omega$; this is possible in view of the boundedness in $L^{\infty}$ of the operator $P_{h}^{0}$ and the assumption (3.12). Moreover, the constant $f_{0}$ denotes $|f(0)|$.

The proof of (3.14) is carried out by induction. In view of $\left(\mathrm{H}_{\beta}\right)_{1},\left(\mathrm{H}_{f}\right)$ and (3.2), the following inequalities hold in $\Omega$ :

$$
\begin{gathered}
\beta\left(-C_{n-1}\right) \leq \beta\left(U^{n-1}\right) \leq \beta\left(C_{n-1}\right), \\
-f_{0}+L_{f} \beta\left(-C_{n-1}\right) \leq f\left(\beta\left(U^{n-1}\right)\right) \leq f_{0}+L_{f} \beta\left(C_{n-1}\right), \\
\alpha\left(-C_{n-1}\right) \leq \alpha\left(U^{n-1}\right) \leq \alpha\left(C_{n-1}\right) .
\end{gathered}
$$

Let $\bar{x} \in \Omega$ be a point at which $\Theta^{n}$ attains its maximum. Since $\Theta^{n}$ is piecewise linear, $\bar{x}$ is clearly a node of $\mathbf{S}_{h}$. Let $\bar{\phi} \in V_{h}^{1}$ be the corresponding shape function. Now, with the aid of the discrete equation (2.11), inequality (3.11), the definition (2.1) of the quadrature rule and the elementary property $\int_{\Omega} \bar{\phi}=|\operatorname{supp} \bar{\phi}| /(d+1)$, we arrive at

$$
\begin{aligned}
\Theta^{n}(\bar{x}) \frac{|\operatorname{supp} \bar{\phi}|}{d+1} & =\left\langle\Theta^{n}, \bar{\phi}\right\rangle_{h} \leq\left\langle\beta\left(U^{n-1}\right), \bar{\phi}\right\rangle+\frac{\tau}{\mu}\left\langle f\left(\beta\left(U^{n-1}\right)\right), \bar{\phi}\right\rangle \\
& \leq\left\{\beta\left(C_{n-1}\right)+\frac{\tau}{\mu}\left[f_{0}+L_{f} \beta\left(C_{n-1}\right)\right]\right\} \frac{|\operatorname{supp} \bar{\phi}|}{d+1}
\end{aligned}
$$


Here we have also made use of (3.15) and (3.16) to obtain the last inequality. The same argument produces a bound from below for $\Theta^{n}$ when arguing with the minimum of $\Theta^{n}$. Therefore, we get the estimate

$$
\beta\left(-C_{n-1}\right)-\frac{\tau}{\mu}\left[f_{0}-L_{f} \beta\left(-C_{n-1}\right)\right] \leq \Theta^{n} \leq \beta\left(C_{n-1}\right)+\frac{\tau}{\mu}\left[f_{0}+L_{f} \beta\left(C_{n-1}\right)\right] .
$$

Now, since

$$
U^{n}=U^{n-1}+\mu\left[P_{h}^{0} \Theta^{n}-\beta\left(U^{n-1}\right)\right]=\alpha\left(U^{n-1}\right)+\mu P_{h}^{0} \Theta^{n},
$$

(3.17) and (3.18) yield

$$
-C_{n-1}-\tau\left[f_{0}-L_{f} \beta\left(-C_{n-1}\right)\right] \leq U^{n} \leq C_{n-1}+\tau\left[f_{0}+L_{f} \beta\left(C_{n-1}\right)\right] \text { in } \Omega .
$$

By $\left(\mathrm{H}_{\beta}\right)_{1}$ we can rewrite this expression as follows,

$$
\begin{aligned}
\left\|U^{n}\right\|_{L^{\infty}(\Omega)} & \leq C_{n-1}+\tau\left[f_{0}+L_{f} \max \left(\beta\left(C_{n-1}\right),-\beta\left(-C_{n-1}\right)\right)\right] \\
& \leq \tau f_{0}+C_{n-1}\left[1+\tau L_{f} L_{\beta}\right] .
\end{aligned}
$$

Finally, the claim (3.14) is an easy consequence of the inequality $1+\tau L_{f} L_{\beta} \leq$ $e^{\tau L_{f} L_{\beta}}$, which leads to

$$
\tau f_{0}+C_{n-1}\left[1+\tau L_{f} L_{\beta}\right] \leq C_{n},
$$

where $C_{n}$ was defined in (3.14).

Remark 6 . It is easily seen from Lemma 2 that the function $\beta$ may be assumed to be only locally Lipschitz continuous, provided that $f=0$ (see also [2], [13]). For instance, this happens for the porous medium equation, for which $\beta(s):=s|s|^{m-1}$ $(m>1)$.

Remark 7. We now consider the particular, but still relevant, case $u_{0} \geq 0$, $f(0)=0$, which includes porous medium equations, as well as one-phase Stefan problems. Then the continuous physical unknowns $u$ and $\theta$ are clearly nonnegative. Since we wish this property to be preserved in our fully discrete scheme, we analyze the sign of the discrete solution. For $\tau>0$ small enough, the function $I+\frac{\tau}{\mu} f$ is nondecreasing. Therefore, assuming by induction that $U^{n-1} \geq 0$ (which holds for $n=1$ because $\left.U^{0}=P_{h}^{0} u_{0} \geq 0\right)$, we get $\left[I+\frac{\tau}{\mu} f\right]\left(\beta\left(U^{n-1}\right)\right) \geq 0$. The facts that the matrix of the linear system (2.11) is an $M$-matrix and the right-hand side is nonnegative imply $\Theta^{n} \geq 0$. Finally, we get $U^{n}=\alpha\left(U^{n-1}\right)+\mu P_{h}^{0} \Theta^{n} \geq 0$, concluding the argument.

4. Error Analysis in Energy Norms. The primary aim of this section is to analyze the accuracy of the fully discrete nonlinear Chernoff formula (2.11), (2.12) in approximating the physical unknowns $\theta$ and $u$. The key ideas were already explained and successfully used by Magenes, Nochetto and Verdi [13] in studying the discrete-time analogue of this algorithm. So the present concern is to extend these ideas to the fully discrete scheme $\left(\mathrm{P}_{h, \tau}\right)$. However, we believe that the current results deserve a detailed presentation.

Before establishing the precise statements, let us show what the general strategy is. We first define the errors $e_{\theta}$ and $e_{u}$ by

$$
e_{\theta}(t):=\theta(t)-\Theta^{n}, \quad e_{u}(t):=u(t)-U^{n} \text { for } t \in I^{n}, 1 \leq n \leq N .
$$


Then, after integrating (2.9) on $I^{n}$, we can write the set of discrete-in-time equations satisfied by the continuous solution; namely,

$$
\left\langle\partial u^{n}, \phi\right\rangle+\left\langle\nabla \bar{\theta}^{n}, \nabla \phi\right\rangle=\left\langle\bar{f}^{n}(\beta(u)), \phi\right\rangle \quad \forall \phi \in H_{0}^{1}(\Omega), 1 \leq n \leq N
$$

We now take the difference between (4.2) and (3.1), sum over $n$ from 1 to $i \leq N$ and multiply by $\tau$. The resulting expression is the following error equation,

$$
\begin{aligned}
\left\langle e_{u}^{i}-e_{u}^{0}, \chi\right\rangle+ & \left\langle\nabla \sum_{n=1}^{i} \tau\left[\bar{\theta}^{n}-\Theta^{n}\right], \nabla \chi\right\rangle \\
= & \mu\left\langle\left[I-P_{h}^{0}\right] \sum_{n=1}^{i} \Theta^{n}, \chi\right\rangle_{h} \\
& +\left\langle\sum_{n=1}^{i} \tau\left[\bar{f}^{n}(\beta(u))-f\left(\beta\left(U^{n-1}\right)\right)\right], \chi\right\rangle \quad \forall \chi \in V_{h}^{1}
\end{aligned}
$$

The next step is to choose a suitable test function $\chi$. Let us take $\chi:=\tau\left[P_{h}^{1} \bar{\theta}^{i}-\Theta^{i}\right] \in$ $V_{h}^{1}$ and sum over $i$ from 1 to $m \leq N$. After reordering we get

$$
\begin{aligned}
& \sum_{i=1}^{m} \int_{I^{i}}\left\langle e_{u}(t), e_{\theta}(t)\right\rangle d t+\sum_{i=1}^{m} \tau^{2}\left\langle\nabla \sum_{n=1}^{i}\left[\bar{\theta}^{n}-\Theta^{n}\right], \nabla\left[P_{h}^{1} \bar{\theta}^{i}-\Theta^{i}\right]\right\rangle=: \mathrm{I}+\mathrm{II} \\
& =\sum_{i=1}^{m} \int_{I^{i}}\left\langle u(t)-u^{i}, e_{\theta}(t)\right\rangle d t+\sum_{i=1}^{m} \tau\left\langle u^{i}-U^{i},\left[I-P_{h}^{1}\right] \bar{\theta}^{i}\right\rangle \\
& \quad+\left\langle u_{0}-U^{0}, \sum_{i=1}^{m} \tau\left[P_{h}^{1} \bar{\theta}^{i}-\Theta^{i}\right]\right\rangle+\mu \sum_{i=1}^{m} \tau\left\langle\left[I-P_{h}^{0}\right] \sum_{n=1}^{i} \Theta^{n}, P_{h}^{1} \bar{\theta}^{i}-\Theta^{i}\right\rangle_{h} \\
& \quad+\sum_{i=1}^{m} \tau\left\langle\sum_{n=1}^{i} \tau\left[\bar{f}^{n}(\beta(u))-f\left(\beta\left(U^{n-1}\right)\right)\right], P_{h}^{1} \bar{\theta}^{i}-\Theta^{i}\right\rangle=: \text { III }+\cdots+\text { VII. }
\end{aligned}
$$

We now proceed to estimate each one of these terms. To begin with, note that

$$
u=\mu \theta+\alpha(u) \text { and } U^{n}=\mu P_{h}^{0} \Theta^{n}+\alpha\left(U^{n-1}\right),
$$

whence

$$
e_{u}(t)=\mu e_{\theta}(t)+\left[\alpha(u(t))-\alpha\left(U^{n-1}\right)\right]+\mu\left[I-P_{h}^{0}\right] \Theta^{n} \quad \text { for } t \in I^{n}
$$

Formula (4.5) is the connection with the phase relaxation scheme [26]. Moreover, we know that

$$
e_{\theta}(t)=\left[\beta(u(t))-\beta\left(U^{n-1}\right)\right]-\frac{1}{\mu}\left[U^{n}-U^{n-1}\right]-\left[I-P_{h}^{0}\right] \Theta^{n} \quad \text { for } t \in I^{n}
$$


Then, in view of (4.6) and (4.7), term I in (4.4) can be split as follows:

$$
\begin{aligned}
\mathrm{I}= & \mu\left\|e_{\theta}\right\|_{L^{2}\left(0, t^{m} ; L^{2}(\Omega)\right)}^{2} \\
& +\sum_{i=1}^{m} \int_{I^{i}}\left\langle\alpha(u(t))-\alpha\left(U^{i-1}\right), \beta(u(t))-\beta\left(U^{i-1}\right)\right\rangle d t \\
& +\frac{\tau}{\mu} \sum_{i=1}^{m}\left\langle U^{i}, U^{i}-U^{i-1}\right\rangle+\sum_{i=1}^{m} \int_{I^{i}}\left\langle e_{\theta}(t), U^{i}-U^{i-1}\right\rangle d t \\
& -\frac{1}{\mu} \sum_{i=1}^{m} \int_{I^{i}}\left\langle u(t), U^{i}-U^{i-1}\right\rangle d t+\sum_{i=1}^{m} \tau\left\langle\left[I-P_{h}^{0}\right] \Theta^{i}, U^{i}-U^{i-1}\right\rangle \\
& -\sum_{i=1}^{m} \int_{I^{i}}\left\langle\alpha(u(t))-\alpha\left(U^{i-1}\right),\left[I-P_{h}^{0}\right] \Theta^{i}\right\rangle d t \\
& +\mu \sum_{i=1}^{m} \int_{I^{i}}\left\langle e_{\theta}(t),\left[I-P_{h}^{0}\right] \Theta^{i}\right\rangle d t=: \mathrm{I}_{1}^{m}+\mathrm{I}_{2}+\cdots+\mathrm{I}_{8} .
\end{aligned}
$$

The fact that both $\alpha$ and $\beta$ are increasing functions implies that $\mathrm{I}_{2} \geq 0$. Term $\mathrm{I}_{3}$ is handled by means of (3.5) and $\left(\mathrm{H}_{u_{0}}\right)$; namely,

$$
\mathrm{I}_{3} \geq \frac{\tau}{2 \mu} \sum_{i=1}^{m}\left[\left\|U^{i}\right\|_{L^{2}(\Omega)}^{2}-\left\|U^{i-1}\right\|_{L^{2}(\Omega)}^{2}\right] \geq-\frac{\tau}{2 \mu}\left\|U^{0}\right\|_{L^{2}(\Omega)}^{2} \geq-C \tau .
$$

For $\mathrm{I}_{4}$ we use inequalities (3.4) and (3.6) to arrive at

$$
\left|\mathrm{I}_{4}\right| \leq \frac{1}{4} \mathrm{I}_{1}^{m}+\tau \sum_{i=1}^{m}\left\|U^{i}-U^{i-1}\right\|_{L^{2}(\Omega)}^{2} \leq \frac{1}{4} \mathrm{I}_{1}^{m}+C \tau .
$$

By virtue of the approximation property (2.5) of $P_{h}^{0}$ and the a priori estimates (3.6) we have

$$
\sum_{i=1}^{m} \tau\left\|\left[I-P_{h}^{0}\right] \Theta^{i}\right\|_{L^{2}(\Omega)}^{2} \leq C h^{2}
$$

Then,

$$
\left|\mathrm{I}_{8}\right| \leq \frac{1}{4} \mathrm{I}_{1}^{m}+C h^{2}
$$

At the same time, using (2.6) and (3.9), we get

$$
\left|\mathrm{I}_{7}\right| \leq C h\left\{\|u\|_{L^{2}\left(0, t^{m} ; L^{2}(\Omega)\right)}+\left[\sum_{i=1}^{m} \tau\left\|U^{i}\right\|_{L^{2}(\Omega)}^{2}\right]^{1 / 2}\right\} \leq C h
$$

and, again by (3.6), we obtain

$$
\left|\mathrm{I}_{6}\right| \leq \tau \sum_{i=1}^{m}\left\|U^{i}-U^{i-1}\right\|_{L^{2}(\Omega)}^{2}+C h^{2} \leq C \tau+C h^{2} .
$$

The remaining term $\mathrm{I}_{5}$ will be analyzed later, under various regularity assumptions on $u_{0}$. Instead, we now bound term II in (4.4). To do so, we need the following elementary identity, which is an easy consequence of $(3.5)$ :

$$
2 \sum_{i=1}^{m} a_{i} \cdot\left[\sum_{n=1}^{i} a_{n}\right]=\left[\sum_{i=1}^{m} a_{i}\right]^{2}+\sum_{i=1}^{m} a_{i}^{2} \quad \text { for } a_{i} \in \mathbf{R}, 1 \leq i \leq N .
$$


Thus, using the approximation property (2.4) of the Ritz projection $P_{h}^{1}$ together with the regularity $\int_{0}^{t} \theta \in L^{\infty}\left(0, T ; H^{2}(\Omega)\right)$, which comes from integrating the original partial differential equation (2.9) in time, we easily obtain

$$
\begin{aligned}
\mathrm{II} & \geq \sum_{i=1}^{m} \tau^{2}\left\langle\nabla \sum_{n=1}^{i}\left[P_{h}^{1} \bar{\theta}^{n}-\Theta^{n}\right], \nabla\left[P_{h}^{1} \bar{\theta}^{i}-\Theta^{i}\right]\right\rangle \\
& \geq \frac{1}{2}\left\|\tau \sum_{i=1}^{m} \nabla\left[P_{h}^{1} \bar{\theta}^{i}-\Theta^{i}\right]\right\|_{L^{2}(\Omega)}^{2} \geq \frac{1}{2}\left\|\nabla \int_{0}^{t^{m}} P_{h}^{1} e_{\theta}(t) d t\right\|_{L^{2}(\Omega)}^{2} \\
& \geq \frac{1}{2}\left\|\nabla \int_{0}^{t^{m}} e_{\theta}(t) d t\right\|_{L^{2}(\Omega)}^{2}-C h^{2}=: \mathrm{II}^{m}-C h^{2} .
\end{aligned}
$$

Treating III requires a duality argument between $H^{-1}(\Omega)$ and $H_{0}^{1}(\Omega)$. The fact that $e_{\theta}$ is uniformly bounded in $H_{0}^{1}(\Omega)$, i.e., $\left\|\nabla e_{\theta}\right\|_{L^{2}\left(0, T ; L^{2}(\Omega)\right)} \leq C$, which results from (2.6) and (3.6), and again the regularity property (2.6), lead to

$$
\begin{aligned}
|\mathrm{III}| & =\left|\sum_{i=1}^{m} \int_{I^{i}}\left\langle-\int_{t}^{t^{i}} \frac{\partial u}{\partial s} d s, e_{\theta}(t)\right\rangle d t\right| \\
& \leq \tau\left\|\frac{\partial u}{\partial t}\right\|_{L^{2}\left(0, t^{m} ; H^{-1}(\Omega)\right)}\left\|e_{\theta}\right\|_{L^{2}\left(0, t^{m} ; H_{0}^{1}(\Omega)\right)} \leq C \tau .
\end{aligned}
$$

The other term IV is analyzed by making use of the approximation property (2.4) as well as (2.6) and (3.9). Indeed, we have

$$
\begin{aligned}
|\mathrm{IV}| \leq & C\left\{\|u\|_{L^{2}\left(0, t^{m} ; L^{2}(\Omega)\right)}+\left[\sum_{i=1}^{m} \tau\left\|U^{i}\right\|_{L^{2}(\Omega)}^{2}\right]^{1 / 2}\right\} \\
& \times\left[\sum_{i=1}^{m} \tau\left\|\left[I-P_{h}^{1}\right] \bar{\theta}^{i}\right\|_{L^{2}(\Omega)}^{2}\right]^{1 / 2} \leq C h .
\end{aligned}
$$

Using now $\left(\mathrm{H}_{u_{0}}\right)$ and the approximation property (2.5), we easily obtain

$$
|\mathrm{V}| \leq C h\left\|u_{0}\right\|_{L^{2}(\Omega)}\left\|\nabla \int_{0}^{t^{m}} P_{h}^{1} e_{\theta}(t) d t\right\|_{L^{2}(\Omega)} \leq \frac{1}{2} \mathrm{II}^{m}+C h^{2} .
$$

In order to get a bound from above for VI, we first rewrite this term in a suitable form; namely,

$$
\begin{aligned}
\mathrm{VI}= & \mu \sum_{i=1}^{m} \tau\left\langle\left[I-P_{h}^{0}\right] \sum_{n=1}^{i} \Theta^{n}, P_{h}^{1} \bar{\theta}^{i}-\Theta^{i}\right\rangle \\
& +\mu \sum_{i=1}^{m} \tau\left[\left\langle\sum_{n=1}^{i} \Theta^{n}, P_{h}^{1} \bar{\theta}^{i}-\Theta^{i}\right\rangle_{h}-\left\langle\sum_{n=1}^{i} \Theta^{n}, P_{h}^{1} \bar{\theta}^{i}-\Theta^{i}\right\rangle\right] \\
= & : \mathrm{VI}_{1}+\mathrm{VI}_{2} .
\end{aligned}
$$

The estimate for $\mathrm{VI}_{1}$ proceeds along the same lines as for III, with the only difference that now we exploit the superconvergence error estimate (2.5). Thus,

$$
\left|\mathrm{VI}_{1}\right| \leq C h^{2} \sum_{i=1}^{m} \tau\left\|\sum_{n=1}^{i} \Theta^{n}\right\|_{H^{1}(\Omega)}\left\|P_{h}^{1} \bar{\theta}^{i}-\Theta^{i}\right\|_{H_{0}^{1}(\Omega)} \leq C \frac{h^{2}}{\tau}
$$


where again the a priori estimates (2.6) and (3.6) have been used. The other term, $\mathrm{VI}_{2}$, is an error due to the quadrature rule; so, with the aid of (2.3), we can control its contribution as follows,

$$
\left|\mathrm{VI}_{2}\right| \leq C h^{2} \sum_{i=1}^{m} \tau\left\|\nabla \sum_{n=1}^{i} \Theta^{n}\right\|_{L^{2}(\Omega)}\left\|\nabla\left[P_{h}^{1} \bar{\theta}^{i}-\Theta^{i}\right]\right\|_{L^{2}(\Omega)} \leq C \frac{h^{2}}{\tau} .
$$

It only remains to estimate the source term VII, which obviously satisfies

$$
\mathrm{VII}=\sum_{i=1}^{m} \int_{I^{i}}\left\langle\sum_{n=1}^{i} \tau\left[\bar{f}^{n}(\beta(u))-f\left(\beta\left(U^{n-1}\right)\right)\right], e_{\theta}(t)+\left[P_{h}^{1}-I\right] \bar{\theta}^{i}\right\rangle .
$$

In view of $(3.4),\left(\mathrm{H}_{f}\right),(4.7)$, the approximation property (2.4) and (4.9), we can write

$$
\begin{aligned}
|\mathrm{VII}| \leq & \frac{1}{4} \mathrm{I}_{1}^{m}+\left\|\left[I-P_{h}^{1}\right] \theta\right\|_{L^{2}\left(0, t^{m} ; L^{2}(\Omega)\right)}^{2} \\
& +C \sum_{i=1}^{m} \tau \sum_{n=1}^{i} \tau\left\|\bar{f}^{n}(\beta(u))-f\left(\beta\left(U^{n-1}\right)\right)\right\|_{L^{2}(\Omega)}^{2} \\
\leq & \frac{1}{4} \mathrm{I}_{1}^{m}+C h^{2}+C \sum_{i=1}^{m} \tau \mathrm{I}_{1}^{i}+C \tau \sum_{i=1}^{m}\left\|U^{i}-U^{i-1}\right\|_{L^{2}(\Omega)}^{2} \\
& +C \sum_{i=1}^{m} \tau\left\|\left[I-P_{h}^{0}\right] \Theta^{i}\right\|_{L^{2}(\Omega)}^{2} \leq C \tau+C h^{2}+\frac{1}{4} \mathrm{I}_{1}^{m}+C \sum_{i=1}^{m} \tau \mathrm{I}_{1}^{i},
\end{aligned}
$$

where we have also employed (2.6) and the discrete $H^{1 / 2}$-bound in (3.6).

Collecting all the previous estimates, and inserting them into (4.4), gives

$$
\begin{aligned}
\frac{\mu}{4}\left\|e_{\theta}\right\|_{L^{2}\left(0, t^{m} ; L^{2}(\Omega)\right)}^{2}+\frac{1}{4}\left\|\nabla \int_{0}^{t^{m}} e_{\theta}(t) d t\right\|_{L^{2}(\Omega)}^{2} \\
\leq C\left[\tau+h+\frac{h^{2}}{\tau}\right]+C \sum_{i=1}^{m} \tau\left\|e_{\theta}\right\|_{L^{2}\left(0, t^{i} ; L^{2}(\Omega)\right)}^{2} \\
+\frac{1}{\mu} \sum_{i=1}^{m} \int_{I^{i}}\left\langle u(t), U^{i}-U^{i-1}\right\rangle d t .
\end{aligned}
$$

The last term will be shown to be $O\left(\tau^{2 \nu}\right)$, where $0<\nu \leq 1 / 2$ depends on the regularity assumed on $u_{0}$ and $\beta\left(u_{0}\right)$ (see the theorems below). Then, applying the discrete Gronwall inequality yields

$$
\left\|e_{\theta}\right\|_{L^{2}(Q)}+\left\|\int_{0}^{t} e_{\theta}\right\|_{L^{\infty}\left(0, T ; H^{1}(\Omega)\right)} \leq C\left[\tau^{\nu}+h^{1 / 2}+\frac{h}{\tau^{1 / 2}}\right]=: \sigma(h, \tau) .
$$

These estimates lead to the following $H^{-1}$-error bound for the unknown $u$,

$$
\left\|e_{u}\right\|_{L^{\infty}\left(0, T ; H^{-1}(\Omega)\right)} \leq C \sigma(h, \tau) .
$$

In order to prove this result, we introduce the Green operator $G: H^{-1}(\Omega) \rightarrow H_{0}^{1}(\Omega)$ associated with the Laplacian; it is defined by

$$
\langle\nabla G \varsigma, \nabla \phi\rangle=\langle\varsigma, \phi\rangle \quad \forall \phi \in H_{0}^{1}(\Omega), \varsigma \in H^{-1}(\Omega) .
$$


The corresponding discrete Green operator $G_{h}: H^{-1}(\Omega) \rightarrow V_{h}^{1}$ is defined by

$$
\left\langle\nabla G_{h} \zeta, \nabla \chi\right\rangle=\langle\varsigma, \chi\rangle \quad \forall \chi \in V_{h}^{1}, \varsigma \in H^{-1}(\Omega) .
$$

These operators enjoy the following properties:

$$
\begin{gathered}
\left\|\nabla G_{h} \zeta\right\|_{L^{2}(\Omega)} \leq\left\|\nabla G_{\zeta}\right\|_{L^{2}(\Omega)}=\|\zeta\|_{H^{-1}(\Omega)}=\langle\zeta, G \zeta\rangle^{1 / 2}, \\
\left\|\left[G-G_{h}\right] \zeta\right\|_{L^{2}(\Omega)} \leq C h^{2}\left\|_{\zeta}\right\|_{L^{2}(\Omega)} .
\end{gathered}
$$

Taking $\chi:=G_{h}\left(u^{i}-U^{i}\right)$ as test function in (4.3) allows one to express the desired $H^{-1}$-bound as follows:

$$
\begin{aligned}
\left\|e_{u}^{i}\right\|_{H^{-1}(\Omega)}^{2}= & \left\|u^{i}-U^{i}\right\|_{H^{-1}(\Omega)}^{2}=\left\langle e_{u}^{i}, G e_{u}^{i}\right\rangle \\
= & \left\langle e_{u}^{i},\left[G-G_{h}\right] e_{u}^{i}\right\rangle+\left\langle\nabla \sum_{n=1}^{i} \tau\left[\Theta^{n}-\bar{\theta}^{n}\right], \nabla G_{h} e_{u}^{i}\right\rangle \\
& +\mu\left\langle\left[I-P_{h}^{0}\right] \sum_{n=1}^{i} \Theta^{n}, G_{h} e_{u}^{i}\right\rangle_{h}+\left\langle e_{u}^{0}, G_{h} e_{u}^{i}\right\rangle \\
& +\left\langle\sum_{n=1}^{i} \tau\left[\bar{f}^{n}(\beta(u))-f\left(\beta\left(U^{n-1}\right)\right)\right], G_{h} e_{u}^{i}\right\rangle=: \text { VIII }+\cdots+\text { XII. }
\end{aligned}
$$

Using the a priori estimates (2.6) and (3.9), together with (4.20), it is easily seen that $|\mathrm{VIII}|=O\left(h^{2}\right)$. In view of $(4.15)$ and (4.19), the next term is bounded by

$$
|\mathrm{IX}| \leq\left\|\nabla G_{h} e_{u}^{i}\right\|_{L^{2}(\Omega)}\left\|\nabla \int_{0}^{t^{i}} e_{\theta}\right\|_{L^{2}(\Omega)} \leq \frac{1}{5}\left\|e_{u}^{i}\right\|_{H^{-1}(\Omega)}^{2}+C \sigma^{2}(h, \tau) .
$$

Arguing as in (4.12) and (4.13) with term $\mathrm{X}$ gives

$$
|\mathrm{X}| \leq C h^{2}\left\|\sum_{n=1}^{i} \Theta^{n}\right\|_{H^{1}(\Omega)}\left\|\nabla G_{h} e_{u}^{i}\right\|_{L^{2}(\Omega)} \leq \frac{1}{5}\left\|e_{u}^{i}\right\|_{H^{-1}(\Omega)}^{2}+C \sigma^{2}(h, \tau),
$$

because we can assume without loss of generality that $\sigma(h, \tau) \leq 1$, thus $h^{2} / \tau \leq 1$. By virtue of $\left(\mathrm{H}_{u_{0}}\right)$ and (2.5), term XI is easily bounded by

$$
|\mathrm{XI}| \leq \frac{1}{5}\left\|e_{u}^{i}\right\|_{H^{-1}(\Omega)}^{2}+C h^{2}
$$

The same technique applied in (4.14) can now be used, together with Poincaré's inequality and (3.6), to arrive at

$$
\begin{aligned}
|\mathrm{XII}| & \leq C\left\|\nabla G_{h} e_{u}^{i}\right\|_{L^{2}(\Omega)} \sum_{n=1}^{i} \int_{I^{n}}\left\|\beta(u(t))-\beta\left(U^{n-1}\right)\right\|_{L^{2}(\Omega)} d t \\
& \leq \frac{1}{5}\left\|e_{u}^{i}\right\|_{H^{-1}(\Omega)}^{2}+C \sigma^{2}(h, \tau) .
\end{aligned}
$$

In conclusion, we have proved the error bound

$$
\max _{1 \leq i \leq N}\left\|u^{i}-U^{i}\right\|_{H^{-1}(\Omega)} \leq C \sigma(h, \tau) .
$$

The assertion (4.16) follows from the fact that

$$
u \in H^{1}\left(0, T ; H^{-1}(\Omega)\right) \subset C^{0,1 / 2}\left(0, T ; H^{-1}(\Omega)\right) .
$$

We are now in a position to state the main result of this paper. 
THEOREM 1. Let $\left(\mathrm{H}_{\Omega}\right),\left(\mathrm{H}_{\beta}\right)_{1},\left(\mathrm{H}_{f}\right)$ and $\left(\mathrm{H}_{\mathbf{S}_{h}}\right)$ hold. Assume that

$$
u_{0} \in L^{\infty}(\Omega), \quad \Delta \beta\left(u_{0}\right) \in L^{1}(\Omega)
$$

and in addition that

$$
\max _{1 \leq n \leq N}\left\|U^{n}\right\|_{L^{\infty}(\Omega)} \leq C .
$$

Let $\tau$ be chosen so that $\tau=C^{*} h$, with $C^{*}$ an arbitrary positive constant. Then we have

$$
\left\|e_{\theta}\right\|_{L^{2}(Q)}+\left\|\int_{0}^{t} e_{\theta}\right\|_{L^{\infty}\left(0, T ; H^{1}(\Omega)\right)}+\left\|e_{u}\right\|_{L^{\infty}\left(0, T ; H^{-1}(\Omega)\right)}=O\left(h^{1 / 2}\right) .
$$

Remark 8. The a priori estimate (4.22) holds, for instance, for triangulations $\mathbf{S}_{h}$ that are of acute type (see Lemma 2).

Remark 9 . The assumption $\Delta \beta\left(u_{0}\right) \in L^{1}(\Omega)$ can be slightly weakened according to Remark 1 , because the only property we need is $\partial u / \partial t \in L^{\infty}(0, T ; M(\Omega))$.

Proof of Theorem 1. In view of estimates (4.15) and (4.16), it remains to demonstrate the bound

$$
\left|\sum_{i=1}^{m} \int_{I^{i}}\left\langle u(t), U^{i}-U^{i-1}\right\rangle d t\right| \leq C \tau .
$$

To this end, we need the following summation by parts formula,

$$
\sum_{i=1}^{m} a_{i}\left[b_{i}-b_{i-1}\right]=a_{m} b_{m}-a_{0} b_{0}-\sum_{i=1}^{m} b_{i-1}\left[a_{i}-a_{i-1}\right] .
$$

We can then rewrite the sum in (4.24) as follows:

$$
\begin{aligned}
& \sum_{i=1}^{m} \int_{I^{i}}\left\langle u(t), U^{i}-U^{i-1}\right\rangle d t=\sum_{i=1}^{m} \tau\left\langle\bar{u}^{i}, U^{i}-U^{i-1}\right\rangle \\
& \quad=\tau\left\langle\bar{u}^{m}, U^{m}\right\rangle-\tau\left\langle u_{0}, U^{0}\right\rangle-\sum_{i=1}^{m} \tau\left\langle\bar{u}^{i}-\bar{u}^{i-1}, U^{i-1}\right\rangle,
\end{aligned}
$$

where $\bar{u}^{0}:=u_{0}$. The assumption $u_{0} \in L^{\infty}(\Omega)$, together with (4.22) and (2.6), implies that the first and middle term are $O(\tau)$. For the last term we note that

$$
\bar{u}^{i}-\bar{u}^{i-1}=\frac{1}{\tau} \int_{I^{i}}[u(t)-u(t-\tau)] d t=\frac{1}{\tau} \int_{I^{i}} \int_{t-\tau}^{t} \frac{\partial u}{\partial s}(s) d s d t
$$

and the fact that $\partial u / \partial t \in L^{\infty}\left(0, T ; M(\Omega)\right.$ ), because $\Delta \beta\left(u_{0}\right) \in L^{1}(\Omega)$ (see (R2) in Subsection 2.2). So, what we would like to do is to use a duality argument between $M(\Omega)$ and $C^{0}(\bar{\Omega})$; unfortunately, this fails, because $U^{i} \notin C^{0}(\bar{\Omega})$. The remedy consists of regularizing $U^{i}$, say by convolution, in such a way that we get continuous functions $U_{\varepsilon}^{i}$ having the properties

$$
\max _{1 \leq i \leq N}\left\|U_{\varepsilon}^{i}\right\|_{L^{\infty}(\Omega)} \leq C, \quad U_{\varepsilon}^{i} \underset{\varepsilon \downarrow 0}{\longrightarrow} U^{i} \text { strongly in } L^{2}(\Omega) .
$$

Thus,

$$
\begin{aligned}
\left|\left\langle\bar{u}^{i}-\bar{u}^{i-1}, U^{i-1}\right\rangle\right| & \leq\left|\left\langle\bar{u}^{i}-\bar{u}^{i-1}, U_{\varepsilon}^{i-1}\right\rangle\right|+\left|\left\langle\bar{u}^{i}-\bar{u}^{i-1}, U^{i-1}-U_{\varepsilon}^{i-1}\right\rangle\right| \\
& \leq C \tau+C\|u\|_{L^{\infty}\left(0, T ; L^{2}(\Omega)\right)}\left\|U^{i-1}-U_{\varepsilon}^{i-1}\right\|_{L^{2}(\Omega)} .
\end{aligned}
$$

Finally, taking the limit as $\varepsilon \downarrow 0$ and using the a priori estimate (2.6) yields the assertion (4.24).

We now extend the previous error estimate to a weaker situation. 
THEOREM 2. Let $\left(\mathrm{H}_{\Omega}\right),\left(\mathrm{H}_{\beta}\right),\left(\mathrm{H}_{f}\right),\left(\mathrm{H}_{u_{0}}\right)$ and $\left(\mathrm{H}_{\mathbf{S}_{h}}\right)$ hold. Let $\tau$ be chosen so that $\tau=C^{*} h^{4 / 3}$ for any positive constant $C^{*}$. Then we have

$$
\left\|e_{\theta}\right\|_{L^{2}(Q)}+\left\|\int_{0}^{t} e_{\theta}\right\|_{L^{\infty}\left(0, T ; H^{1}(\Omega)\right)}+\left\|e_{u}\right\|_{L^{\infty}\left(0, T ; H^{-1}(\Omega)\right)}=O\left(h^{1 / 3}\right) .
$$

Proof. The present task is that of proving the estimate

$$
\left|\sum_{i=1}^{m} \tau\left\langle\bar{u}^{i}, U^{i}-U^{i-1}\right\rangle\right| \leq C \tau^{1 / 2},
$$

which implies that $\nu=1 / 4$ in (4.15). Indeed, the relation between $\tau$ and $h$, as well as the final rate of convergence, are trivial consequences of (4.15) and (4.16). In order to derive (4.26), we make use of the a priori estimates (2.6) and (3.6), which lead to

$$
\begin{aligned}
\left|\sum_{i=1}^{m} \tau\left\langle\bar{u}^{i}, U^{i}-U^{i-1}\right\rangle\right| & \leq C \tau^{1 / 2}\|u\|_{L^{2}\left(0, T ; L^{2}(\Omega)\right)}\left[\sum_{i=1}^{m}\left\|U^{i}-U^{i-1}\right\|_{L^{2}(\Omega)}^{2}\right]^{1 / 2} \\
& \leq C \tau^{1 / 2}
\end{aligned}
$$

and complete the proof.

As one can easily check, the dominating terms on the right-hand side of (4.15) are $\tau^{1 / 4}$ and $h / \tau^{1 / 2}$, rather than $h^{1 / 2}$. If we are interested in getting a better balance between these terms, we can simply modify the discrete scheme as follows. We now look for functions $U^{n} \in V_{h}^{0}$ and $\Theta^{n} \in V_{h}^{1}(1 \leq n \leq N)$ so that for all $\chi \in V_{h}^{1}$ we have

$$
\begin{gathered}
U^{0}:=P_{h}^{0} u_{0}, \quad \Theta^{0}:=P_{h}^{0} \beta\left(u_{0}\right), \\
\left\langle\Theta^{n}, \chi\right\rangle_{h}+\frac{\tau}{\mu}\left\langle\nabla \Theta^{n}, \nabla \chi\right\rangle=\left\langle\beta\left(U^{n-1}\right)+\frac{\tau}{\mu} f\left(\beta\left(U^{n-1}\right)\right), \chi\right\rangle \\
+\left\langle\left[I-P_{h}^{0}\right] \Theta^{n-1}, \chi\right\rangle_{h}, \\
U^{n}:=U^{n-1}+\mu\left[P_{h}^{0} \Theta^{n}-\beta\left(U^{n-1}\right)\right] .
\end{gathered}
$$

This scheme is still stable and computationally feasible. Indeed, the proof follows the same lines as that of Lemma 1, except for the term in (3.8), which is replaced by

$$
\begin{aligned}
& \mu \sum_{n=1}^{m}\left\langle\left[P_{h}^{0}-I\right]\left[\Theta^{n}-\Theta^{n-1}\right], \Theta^{n}\right\rangle_{h}=\mu \sum_{n=1}^{m}\left\langle\left[P_{h}^{0}-I\right]\left[\Theta^{n}-\Theta^{n-1}\right],\left[I-P_{h}^{0}\right] \Theta^{n}\right\rangle_{h} \\
&=- \frac{\mu}{2}\left\langle\left[P_{h}^{0}-I\right] \Theta^{m},\left[P_{h}^{0}-I\right] \Theta^{m}\right\rangle_{h}+\frac{\mu}{2}\left\langle\left[P_{h}^{0}-I\right] \Theta^{0},\left[P_{h}^{0}-I\right] \Theta^{0}\right\rangle_{h} \\
&-\frac{\mu}{2} \sum_{n=1}^{m}\left\langle\left[P_{h}^{0}-I\right]\left[\Theta^{n}-\Theta^{n-1}\right],\left[P_{h}^{0}-I\right]\left[\Theta^{n}-\Theta^{n-1}\right]\right\rangle_{h} \leq 0,
\end{aligned}
$$

where we have used the identity (3.5).

Finally, we have the following improved result, which reproduces for the fully discrete Chernoff approximation the one already shown by Verdi and Visintin for the phase relaxation scheme [26]. 
THEOREM 3. Let $\left(\mathrm{H}_{\Omega}\right),\left(\mathrm{H}_{\beta}\right),\left(\mathrm{H}_{f}\right),\left(\mathrm{H}_{u_{0}}\right)$ and $\left(\mathrm{H}_{\mathbf{S}_{h}}\right)$ hold. Let $\left\{\Theta^{n}, U^{n}\right\}_{n=1}^{N}$ stand for the discrete solutions associated with the scheme (4.27). Then, for $\tau=$ $C^{*} h^{2}$, where $C^{*}$ denotes any positive constant, we get

$$
\left\|e_{\theta}\right\|_{L^{2}(Q)}+\left\|\int_{0}^{t} e_{\theta}\right\|_{L^{\infty}\left(0, T ; H^{1}(\Omega)\right)}+\left\|e_{u}\right\|_{L^{\infty}\left(0, T ; H^{-1}(\Omega)\right)}=O\left(h^{1 / 2}\right) .
$$

This result improves the one in (4.25), but at the expense of a more restrictive time step.

Proof of Theorem 3. We only have to estimate the contribution coming from term VI in (4.4), which now is

$$
\begin{aligned}
\mathrm{VI}= & \mu \sum_{i=1}^{m} \tau\left\langle\left[I-P_{h}^{0}\right] \sum_{n=1}^{i}\left[\Theta^{n}-\Theta^{n-1}\right], P_{h}^{1} \bar{\theta}^{i}-\Theta^{i}\right\rangle_{h} \\
= & \mu \sum_{i=1}^{m} \tau\left\langle\left[I-P_{h}^{0}\right] \Theta^{i}, P_{h}^{1} \bar{\theta}^{i}-\Theta^{i}\right\rangle \\
& +\mu \sum_{i=1}^{m} \tau\left[\left\langle\Theta^{i}, P_{h}^{1} \bar{\theta}^{i}-\Theta^{i}\right\rangle_{h}-\left\langle\Theta^{i}, P_{h}^{1} \bar{\theta}^{i}-\Theta^{i}\right\rangle\right]=: \mathrm{VI}_{1}+\mathrm{VI}_{2}
\end{aligned}
$$

Then, arguing similarly as in (4.12) and (4.13), yields

$$
\left|\mathrm{VI}_{1}\right|,\left|\mathrm{VI}_{2}\right| \leq C h^{2} \sum_{i=1}^{m} \tau\left\|\Theta^{i}\right\|_{H^{1}(\Omega)}\left\|P_{h}^{1} \bar{\theta}^{i}-\Theta^{i}\right\|_{H_{0}^{1}(\Omega)} \leq C h^{2},
$$

which implies the desired result.

We conclude this section with some comments.

Remark 10. From Eq. (2.12) and the a priori estimate (3.6) it follows that

$$
\begin{aligned}
& \sum_{n=1}^{N} \tau\left\|P_{h}^{0} \Theta^{n}-\beta\left(U^{n}\right)\right\|_{L^{2}(\Omega)}^{2} \\
& \quad=\sum_{n=1}^{N} \tau\left\|\frac{1}{\mu}\left[U^{n}-U^{n-1}\right]-\left[\beta\left(U^{n}\right)-\beta\left(U^{n-1}\right)\right]\right\|_{L^{2}(\Omega)}^{2} \leq C \tau .
\end{aligned}
$$

By using (4.9) we get $\left\|e_{\beta(u)}\right\|_{L^{2}(Q)} \leq C \sigma(h, \tau)$, where $e_{\beta(u)}(t):=\theta(t)-\beta\left(U^{n}\right)$ for $t \in I^{n}$.

Remark 11. The error estimates shown above hold also in other situations. Indeed, assume that $\partial \Omega \in C^{1,1}$ ( $\Omega$ is no longer convex!) and that either a nonhomogeneous Dirichlet condition or a linear flux condition is imposed on $\partial \Omega$, i.e.,

$$
\frac{\partial}{\partial \nu} \beta(u)+p(x) \beta(u)=g(x, t) \quad \text { on } \partial \Omega
$$

where $0 \leq p(x) \leq P<\infty$ for a.e. $x \in \partial \Omega$. In this case, the domain cannot be decomposed exactly, and one is forced to analyze the layer $\Omega \Delta \Omega_{h}$. The appropriate techniques to handle such a case were presented by Nochetto and Verdi in [20] and are omitted here.

Remark 12. Assume that a mixed boundary condition is imposed on the boundary. Thus, there is a lack of regularity, which makes the standard $L^{2}$-duality argument fail. However, the error bound $\left\|\left[I-P_{h}^{1}\right] z\right\|_{L^{2}(\Omega)} \leq C_{\delta} h^{1 / 2-\delta}\|z\|_{H_{0}^{1}(\Omega)}$ still holds. The current analysis applies in this case, provided the surface of separation 
between Dirichlet and Neumann conditions is regular enough, giving in the weakest situation of Theorem 2 a rate of convergence $O\left(h^{1 / 4-\delta}\right)$ under the mild relation $\tau=C^{*} h$.

5. Numerical Results. In this section we first describe the implementation of our scheme and then the results of some numerical tests. These experiments were performed in collaboration with M. Paolini and G. Sacchi. Further discussions, comparing performances of the present algorithm and other techniques in approximating both solutions and interfaces, will appear elsewhere, [21].

5.1. Implementation of the Scheme. To begin with, we rewrite the discrete problem in matrix form. Denoting by $\left\{\phi^{j}\right\}_{j=1}^{J}$ and $\left\{\psi^{k}\right\}_{k=1}^{K}$ the canonical basis of $V_{h}^{1}$ and $V_{h}^{0}$, respectively, we define the following matrices

$$
\begin{gathered}
\mathbf{M}:=\left\{\left\langle\phi^{j}, \phi^{l}\right\rangle_{h}\right\}_{j, l=1}^{J}, \quad \mathbf{K}:=\left\{\left\langle\nabla \phi^{j}, \nabla \phi^{l}\right\rangle\right\}_{j, l=1}^{J}, \quad \mathbf{A}:=\mathbf{M}+\frac{\tau}{\mu} \mathbf{K}, \\
\mathbf{P}:=\left\{\left\langle\phi^{j}, \psi^{k}\right\rangle\right\}_{j=1}^{J}, \underset{k=1}{K} .
\end{gathered}
$$

Moreover, we denote by $y_{k}$ the barycenter of $S_{k} \in \mathbf{S}_{h}$ and by $\left\{x_{j}\right\}_{j=1}^{J}$ the nodes of $\mathbf{S}_{h}$. For any $\phi \in V_{h}^{1}$ and $\psi \in V_{h}^{0}$ we also denote by $\boldsymbol{\Phi}=\left\{\phi_{j}\right\}_{j=1}^{J}$ and $\boldsymbol{\Psi}=\left\{\psi_{k}\right\}_{k=1}^{K}$ the vectors with the nodal values of $\phi$ and the barycentric values of $\psi$, respectively. Setting

$$
B^{n-1}:=\sum_{k=1}^{K} \beta\left(U_{k}^{n-1}\right) \psi^{k} \quad \text { and } \quad F^{n-1}:=\sum_{k=1}^{K} f\left(\beta\left(U_{k}^{n-1}\right)\right) \psi^{k},
$$

and noting that $P_{h}^{0} \phi=\sum_{k=1}^{K} \phi\left(y_{k}\right) \psi^{k}$ for $\phi \in V_{h}^{1}$, the discrete problem $\left(\mathrm{P}_{h, \tau}\right)$ can be written equivalently as follows:

$$
\begin{gathered}
\mathbf{A} \Theta^{n}=\mathbf{P}\left[\mathbf{B}^{n-1}+\frac{\tau}{\mu} \mathbf{F}^{n-1}\right], \\
U_{k}^{n}=U_{k}^{n-1}+\mu\left[\Theta^{n}\left(y_{k}\right)-\beta\left(U_{k}^{n-1}\right)\right], \quad k=1, \ldots, K .
\end{gathered}
$$

Since $\mathbf{A}$ is a symmetric and positive definite matrix, the linear system (5.1) admits one and only one solution. The crucial point in the performance of the method is the efficient resolution of the linear system (5.1), because Eq. (5.2) is just a set of scalar algebraic corrections.

Our algorithm has been implemented for general polygonal domains in two dimensions by using an automatic grid code to decompose the domain into triangles. A Cholesky factorization of the matrix $\mathbf{A}$ is recommended whenever the bandwidth is "small" [1]. Indeed, since the matrix $\mathbf{A}$ remains unchanged at each time step, the factorization is made only once at the beginning. Nevertheless, when the use of an automatic grid code is required, for instance in decomposing a general domain, the bandwidth might be "large." Then, iterative methods seem to perform better than direct ones. In particular, since $\mathbf{A}$ is positive definite, an incomplete Cholesky factorization for preconditioning the matrix $A$, combined with conjugate gradient iterations, is recommended for solving the linear system (5.1) [1], [15], [21]. Moreover, the matrix $A$ is strictly diagonally dominant in the experiments below, which makes the preconditioning easier [1], [15], [21]. 
We now turn our attention to the approximation of the free boundary. The location of this crucial unknown as zero-level set of the solution $\left\{\Theta^{n}\right\}_{n=1}^{N}$ is inaccurate for two-phase problems and simply impossible for one-phase problems. This is due to the behavior of the operator $\left[I-\frac{\tau}{\mu} \Delta\right]^{-1}$, which smooths out singularities or, in other words, which replaces a movement of the interface at finite velocity by a diffusion at infinite speed. Moreover, the higher the interface velocity is, the bigger is the artificial diffusion added by $\left[I-\frac{\tau}{\mu} \Delta\right]^{-1}$. This heuristic explanation tells us two things. First, we have to seek the discrete interface as a $\sigma$-level set for $\sigma \neq 0$; this is in the spirit of [19]. Second, the level $\sigma$ should be related to the expected speed of the free boundary, rather than being kept fixed.

5.2. Numerical Tests. The goals of our numerical experiments were to evaluate the actual order of convergence of both variables $u$ and $\theta$, as well as the approximation of the free boundary. If $U^{n}$ and $\Theta^{n}$ are the discrete solutions associated with a mesh size $h$, we set

$$
E_{\theta}^{h}:=\left\{\tau \sum_{n=1}^{N} \sum_{k=1}^{K} \int_{S_{k}} \Pi_{h}\left(\left[\Theta^{n}-\theta^{n}\right]^{2}\right) d x\right\}^{1 / 2}
$$

and analogously for $E_{u}^{h}$, with obvious changes where $u$ is discontinuous. Then, assuming the relations $E_{u}^{h}=C h^{p_{u}}$ and $E_{\theta}^{h}=C h^{p_{\theta}}$, it follows that

$$
p_{u}=\frac{\log \left(E_{u}^{h_{1}} / E_{u}^{h_{2}}\right)}{\log \left(h_{1} / h_{2}\right)}, \quad p_{\theta}=\frac{\log \left(E_{\theta}^{h_{1}} / E_{\theta}^{h_{2}}\right)}{\log \left(h_{1} / h_{2}\right)} .
$$

The space domains in all examples below are rectangles, which are decomposed uniformly. The discrete initial data are chosen according to Remark 2. Examples $1,2,3$ are concerned with the two-phase Stefan problem for which the function $\beta$ is

$$
\begin{gathered}
\beta(u):=c_{1} u \quad \text { if } u<0, \quad \beta(u):=0 \quad \text { if } 0 \leq u \leq 1, \\
\beta(u):=c_{2}(u-1) \quad \text { if } u>1 .
\end{gathered}
$$

Example 4 is the Barenblatt-Pattle solution of the porous medium equation for $\beta(u):=u|u|$. We also point out that, even if mixed boundary conditions are prescribed in our examples, both the approximation property (2.4) and the error estimates (4.23), still hold; indeed, the regularity of the associated Green operator follows from applying reflection techniques. All experiments were performed on an IBM 4361, using the relation $\tau=C^{*} h$.

Notations. N: number of time steps, Nel: number of triangles, Nn: number of nodes, CPU: CPU time in seconds.

Example 1 ([2], [20], [24]). Let $\Omega=(0,0.5) \times(0,0.25), 0<t<T:=0.25$, $C_{1}=C_{2}=1$. The exact enthalpy is

$$
u(x, y, t):= \begin{cases}2\left[e^{\Phi(x, y, t)}-1\right]+1 & \text { if } \Phi(x, y, t) \geq 0 \\ e^{\Phi(x, y, t)}-1 & \text { if } \Phi(x, y, t)<0\end{cases}
$$

where $\Phi(x, y, t):=-x-y+2 t+0.1=0$ is the free boundary. Neumann conditions are assigned on the sides $x=0.5$ and $y=0.25$, and Dirichlet data are prescribed on the sides $x=0$ and $y=0$. We obtained the results summarized in Table 1 and Figures 1 and 2 . 
TABLE 1

\begin{tabular}{rrrcccrr}
\hline Nel & $\mathrm{Nn}$ & $\mathrm{N}$ & $E_{u} \cdot 10^{2}$ & $E_{\theta} \cdot 10^{3}$ & $p_{u}$ & $p_{\theta}$ & $\mathrm{CPU}$ \\
\hline 100 & 66 & 25 & 5.29 & 5.89 & & & 9 \\
196 & 120 & 35 & 4.99 & 4.77 & & & 30 \\
400 & 231 & 50 & 4.48 & 3.88 & & & 107 \\
900 & 496 & 75 & 4.05 & 3.06 & & & 457 \\
\hline
\end{tabular}

Example $2([20],[24])$. Let $\Omega:=(-0.22,0.18) \times(0,0.2), 0<t<T:=0.4$, $C_{1}=1 / 2, C_{2}=1 / 3$. The exact enthalpy is

$$
u(x, y, t):= \begin{cases}6 \Phi(x, y, t)+1 & \text { if } \Phi(x, y, t) \geq 0 \\ 2 \Phi(x, y, t) & \text { if } \Phi(x, y, t)<0\end{cases}
$$

where $\Phi(x, y, t):=x^{2}+y^{2}-e^{-4 t} / 4 e^{2.4}=0$ is the interface. Dirichlet data are assigned on the sides $x=-0.22, x=0.18$ and $y=0.2$, and a vanishing flux condition is prescribed on $x=0$. Table 2 contains the computational results. Discrete and continuous isothermal curves are shown in Figures 3-5.

TABle 2

\begin{tabular}{rrrccccr}
\hline Nel & $\mathrm{Nn}$ & $\mathrm{N}$ & $E_{u} \cdot 10^{2}$ & $E_{\theta} \cdot 10^{4}$ & $p_{u}$ & $p_{\theta}$ & $\mathrm{CPU}$ \\
\hline 64 & 45 & 20 & 5.51 & 12.46 & & & 4 \\
& & & & & 0.28 & 0.44 & \\
144 & 91 & 30 & 4.93 & 10.43 & & & 16 \\
& & & & & 0.16 & 0.52 & \\
324 & 190 & 45 & 4.62 & 8.46 & & & 67 \\
784 & 435 & 70 & 4.06 & 7.00 & & & 328 \\
\hline
\end{tabular}

Example $3([20],[26])$. Let $\Omega:=(0,0.85) \times(0,0.1), 0<t<T:=1, C_{1}=C_{2}=1$. The exact enthalpy $u(x, y, t)$, which does not depend on $y$, is defined by

$$
\begin{aligned}
& \left\{\begin{array}{ll}
4\left[s_{2}(t)-x\right] & \text { if } x \leq s_{2}(t), \\
-\left[x-s_{2}(t)\right]^{2} & \text { if } x>s_{2}(t),
\end{array} \quad \text { if } 0<t \leq 0.25 ;\right.
\end{aligned}
$$

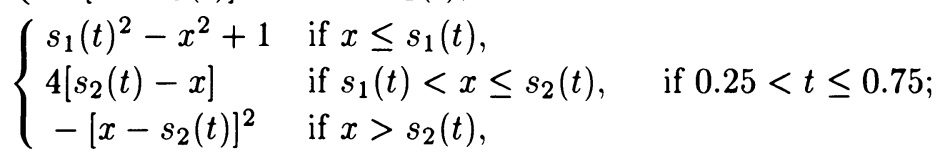

$$
\begin{aligned}
& \left\{\begin{array}{ll}
s_{1}(t)^{2}-x^{2}+1 & \text { if } x \leq s_{1}(t), \\
-\left[x-s_{1}(t)\right]^{2}-2\left[x-s_{1}(t)\right][t-0.75] & \text { if } x>s_{1}(t),
\end{array} \quad \text { if } 0.75<t<1,\right.
\end{aligned}
$$

where $s_{1}(t):=t-0.25$ and $s_{2}(t):=0.5[t+0.25]$ determine the mushy region. A Dirichlet condition is assigned on $x=0.85$ and homogeneous Neumann conditions are imposed on the sides $x=0$ and $y=0, y=0.1$. We obtained the results contained in Table 3. 
TABLE 3

\begin{tabular}{rrrcccrr}
\hline Nel & $\mathrm{Nn}$ & $\mathrm{N}$ & $E_{u} \cdot 10^{2}$ & $E_{\theta} \cdot 10^{3}$ & $p_{u}$ & $p_{\theta}$ & $\mathrm{CPU}$ \\
\hline 24 & 26 & 31 & 5.38 & 8.30 & & & 4 \\
& & & & & 0.35 & 0.79 & \\
96 & 75 & 62 & 4.22 & 4.81 & & & 42 \\
& & & & & 0.33 & 0.78 & \\
384 & 245 & 124 & 3.35 & 2.81 & & & 439 \\
& & & & & 0.31 & 0.75 & \\
1536 & 873 & 248 & 2.69 & 1.66 & & & 4581 \\
\hline
\end{tabular}

The evolution in time of discrete and continuous free boundaries is shown in Figure 6, whereas discrete enthalpies for several time steps are drawn in Figure 7 as functions of the space variable.

Example 4. Let $\Omega:=(2,3) \times(0,0.1), 0<t<T:=1$. The exact density is

$$
u(x, y, t):= \begin{cases}\frac{1}{s(t)}\left[1-\left(\frac{x}{s(t)}\right)^{2}\right] & \text { if } x<s(t) \\ 0 & \text { if } x \geq s(t),\end{cases}
$$

where $s(t)=[12(t+1)]^{1 / 3}$ determines the interface. The solution does not depend on $y$; hence, we prescribe a vanishing flux on $y=0$ and $y=0.1$. Dirichlet data are imposed on $x=2$ and $x=3$. Table 4 sums up the computational results.

TABLE 4

\begin{tabular}{rrrrcrrr}
\hline Nel & $\mathrm{Nn}$ & $\mathrm{N}$ & $E_{u} \cdot 10^{3}$ & $E_{\theta} \cdot 10^{4}$ & $p_{u}$ & $p_{\theta}$ & $\mathrm{CPU}$ \\
\hline 40 & 33 & 20 & 6.97 & 8.43 & & & 7 \\
90 & 64 & 30 & 5.51 & 6.36 & & & 26 \\
250 & 156 & 50 & 3.92 & 4.38 & & & 162 \\
640 & 369 & 80 & 2.84 & 2.93 & & & 825 \\
\hline
\end{tabular}

Figures 8 and 9 illustrate the evolution of the true and approximate interfaces as well as of the density $u$ for various time steps.

The actual rates of convergence of the variable $\theta$ agree with the theoretical one shown in Theorem 1. Moreover, the algorithm seems to approximate the variable $u$ in $L^{2}(Q)$ as well. Even though this is not suggested by theoretical results, the observed rate is almost $O\left(h^{1 / 4}\right)$ for the Stefan problem.

The numerical evidence indicates that the present algorithm is not capable of locating interfaces without any further treatment of the discrete solution; this is due to the artificial diffusion added by the operator $\left[I-\frac{\tau}{\mu} \Delta\right]^{-1}$. Since the location of level sets away from the free boundary is quite precise, the correct remedy might be a sort of local postprocessing of the discrete solution; this is the subject of current research. However, problems having a smooth free boundary which moves along a prescribed direction suggested by the initial datum can be simply handled as follows. The discrete free boundary at time $t^{n}$ is to be sought as a $\sigma_{n}$-level 
set of the variable $\Theta^{n}$, where $\sigma_{n}$ depends on the discrete interface velocity. More precisely, we propose the empirical relation

$$
\sigma_{n}:=C h^{1 / 2} \quad \text { for } n=1,2 ; \quad \sigma_{n}:=\frac{\partial s^{n-1}}{\partial s^{n-2}} \sigma_{n-1} \quad \text { for } 3 \leq n \leq N,
$$

where $\partial s^{n}$ denotes the average change of the discrete interface location from time $t^{n-1}$ to $t^{n}$ and $C>0$ is a constant to be determined in dependence of the initial velocity.

We finally present some pictures illustrating the success of the above procedure in approximating the exact free boundaries of our four numerical tests. Discrete and continuous isothermal curves are depicted as well.

Example 1. $\mathrm{Nel}=900, \mathrm{Nn}=496, \mathrm{~N}=75 ; h=2.357 \times 10^{-2}, \tau=3.333 \times 10^{-3}$, $\sigma_{1}=\sigma_{2}=3.93 \times 10^{-2}$.

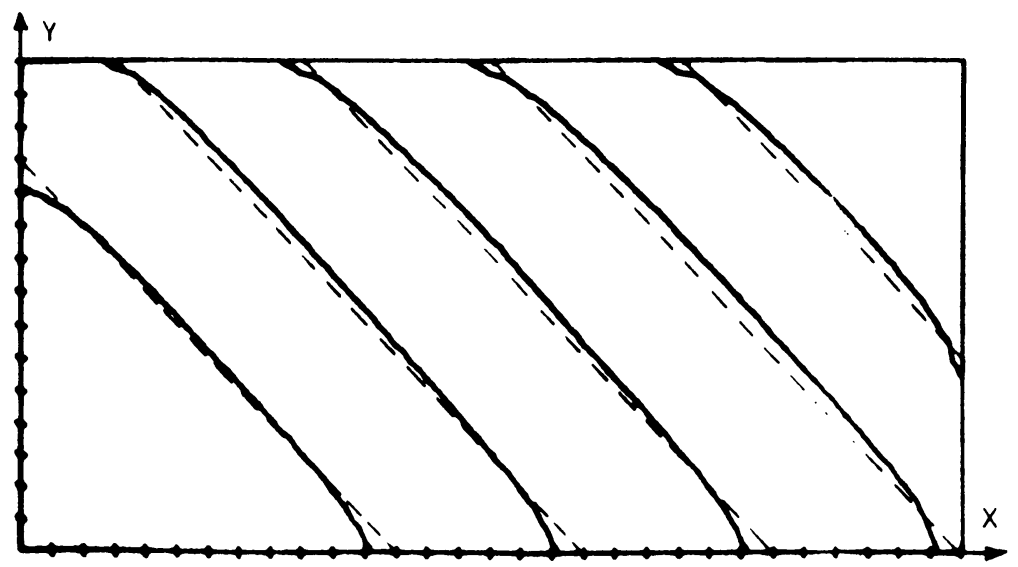

FIGURE 1

Discrete and continuous (dotted lines) interfaces at times $t=0.05,0.1,0.15,0.2,0.25$.

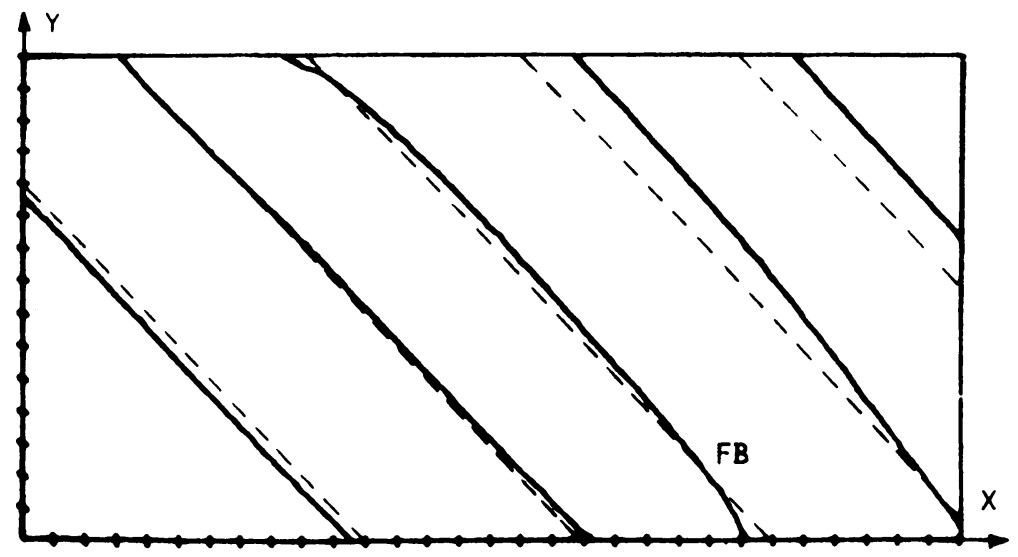

FIGURE 2

Discrete and continuous (dotted lines) interfaces and $\sigma$-isothermal curves for $t=0.15$ and $\sigma=-0.1,-0.2$, $0.2,0.5$. 
Example 2. $\mathrm{Nel}=784, \mathrm{Nn}=435, \mathrm{~N}=70 ; h=2.02 \times 10^{-2}, \tau=5.71 \times 10^{-3}$, $\sigma_{1}=\sigma_{2}=2.02 \times 10^{-2}$.
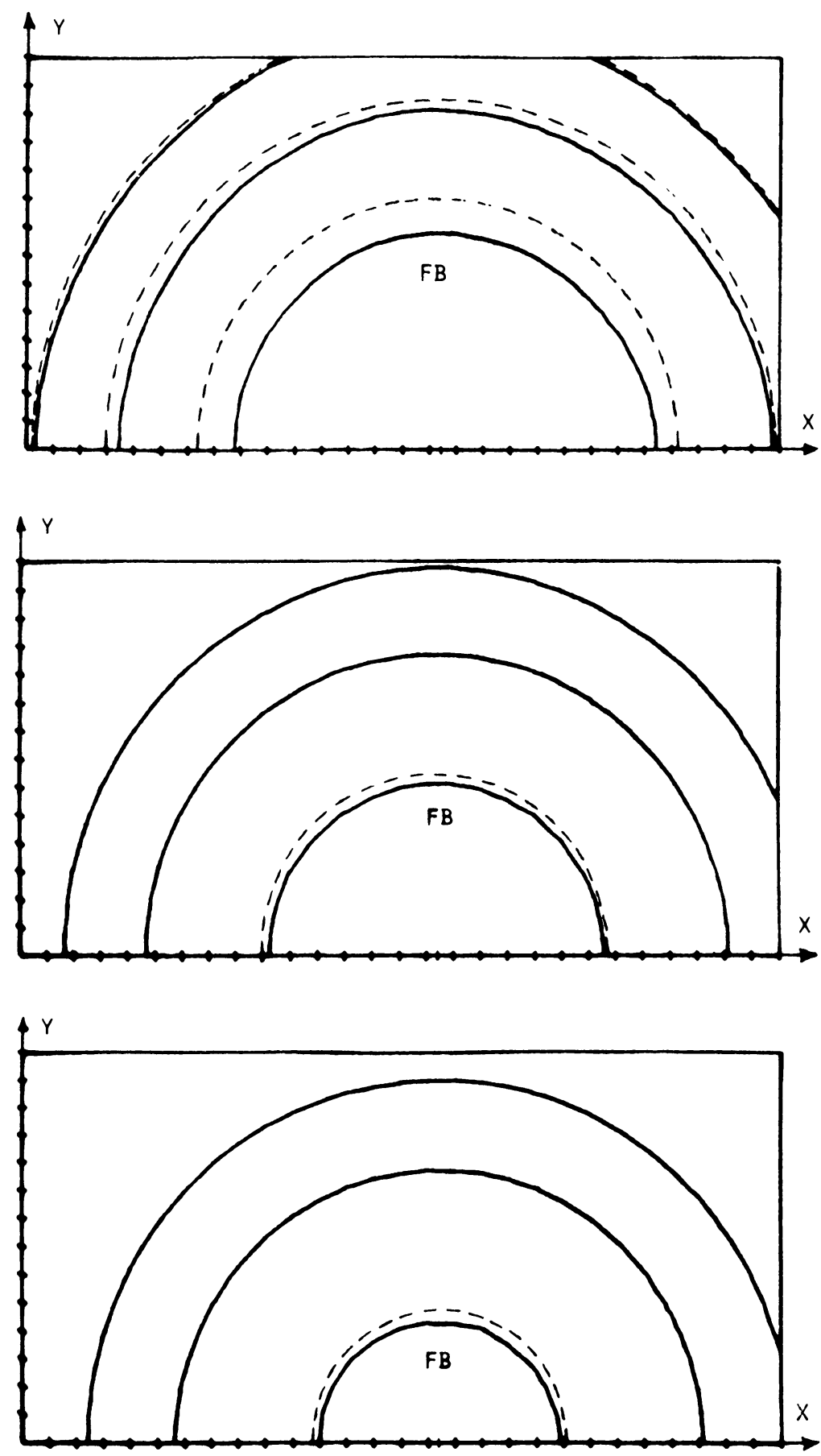

FIGURES 3-5

Discrete and continuous (dotted lines) interfaces and $\sigma$-isothermal curves for $t=0.08,0.24,0.4$ and $\sigma=0.03,0.06$. 
Example 3. $\mathrm{Nel}=1536, \mathrm{Nn}=873, \mathrm{~N}=248 ; h=1.53 \times 10^{-2}, \tau=4.03 \times 10^{-3}$, $\sigma_{1}^{1}=\sigma_{2}^{1}=2.04 \times 10^{-2}, \sigma_{1}^{2}=\sigma_{2}^{2}=0$.

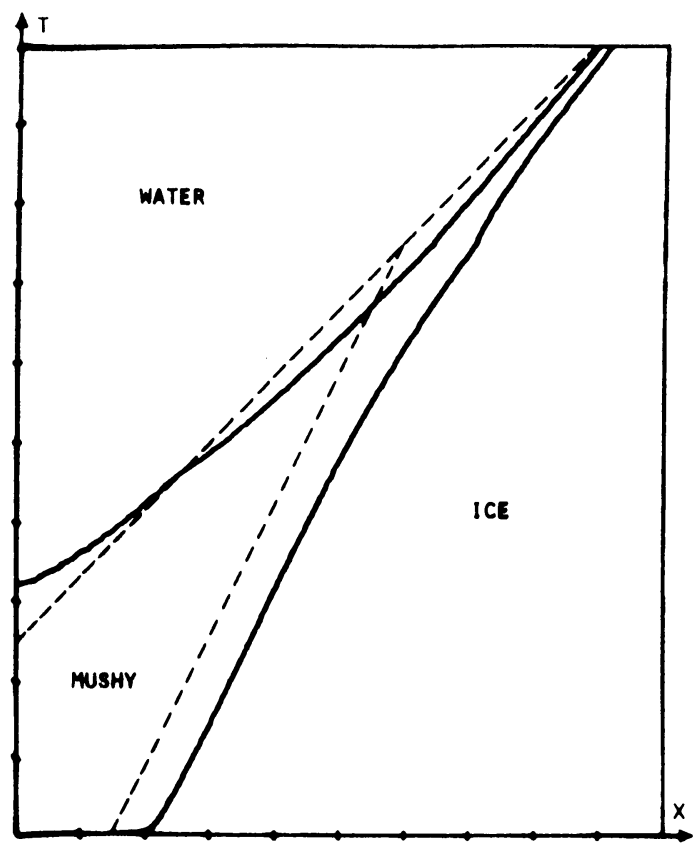

FIGURE 6

Discrete and continuous (dotted lines) interfaces for $y=$ constant.

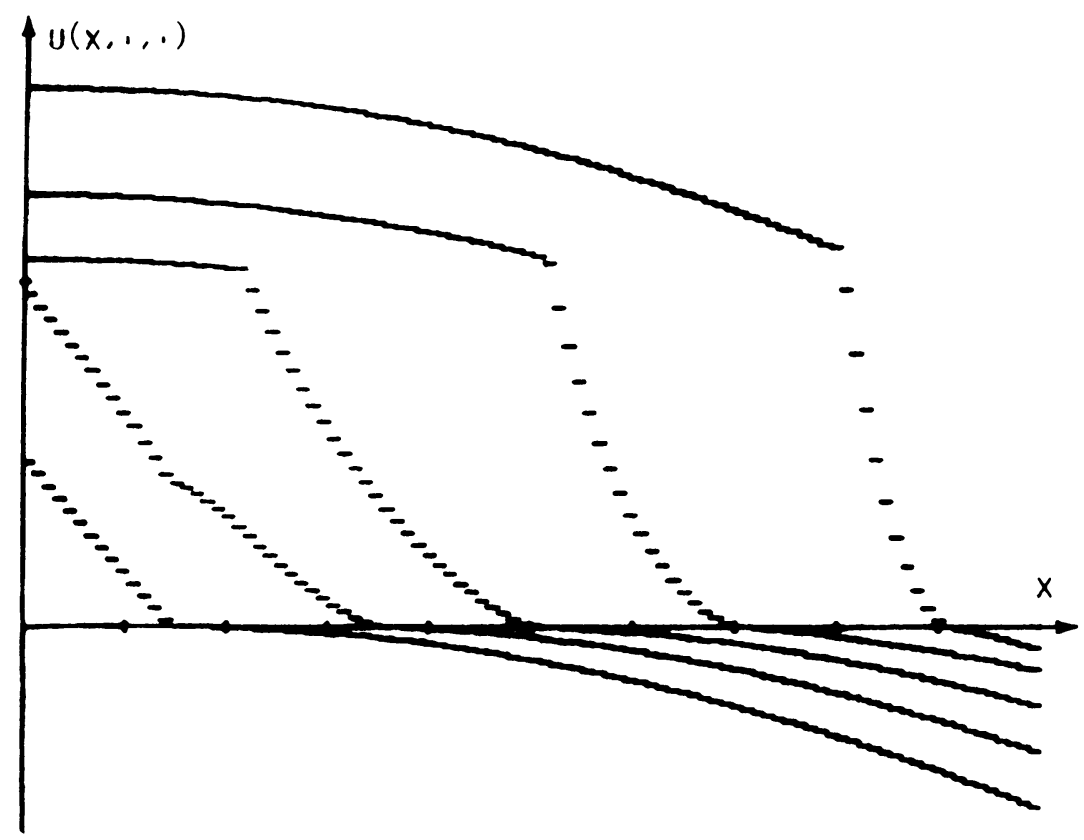

FIGURE 7

Discrete enthalpy for $t=0 ., 0.25,0.5,0.75,1$. and $y=$ constant . 
Example 4. $\mathrm{Nel}=640, \mathrm{Nn}=369, \mathrm{~N}=80 ; h=2.79 \times 10^{-2}, \tau=1.25 \times 10^{-2}$, $\sigma_{1}=\sigma_{2}=2.54 \times 10^{-3}$.

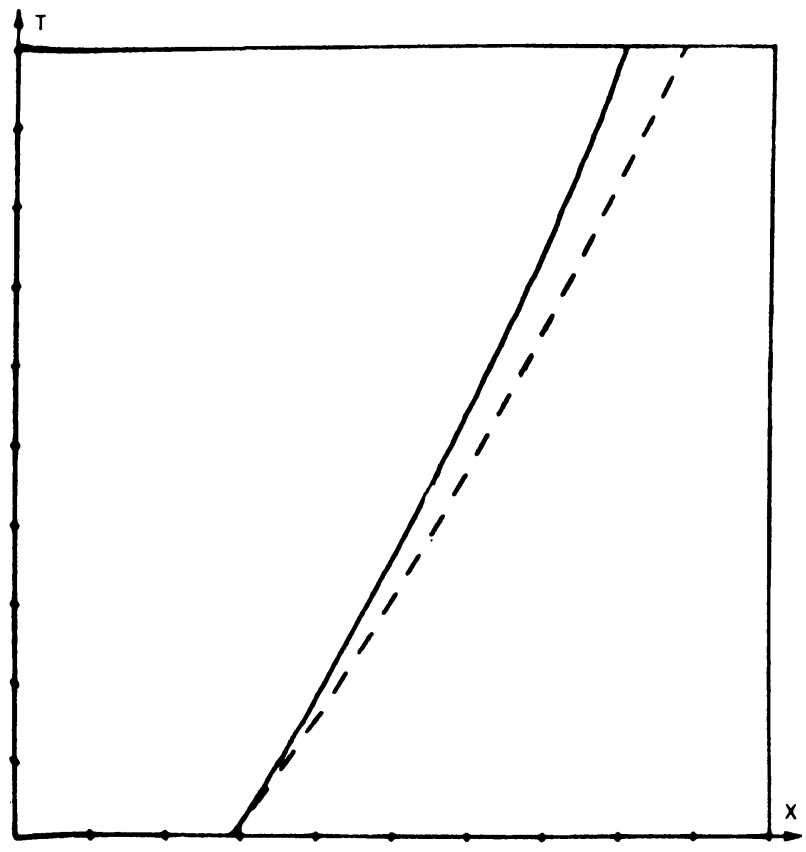

FIGURE 8

Discrete and continuous (dotted lines) interfaces for $y=$ constant .

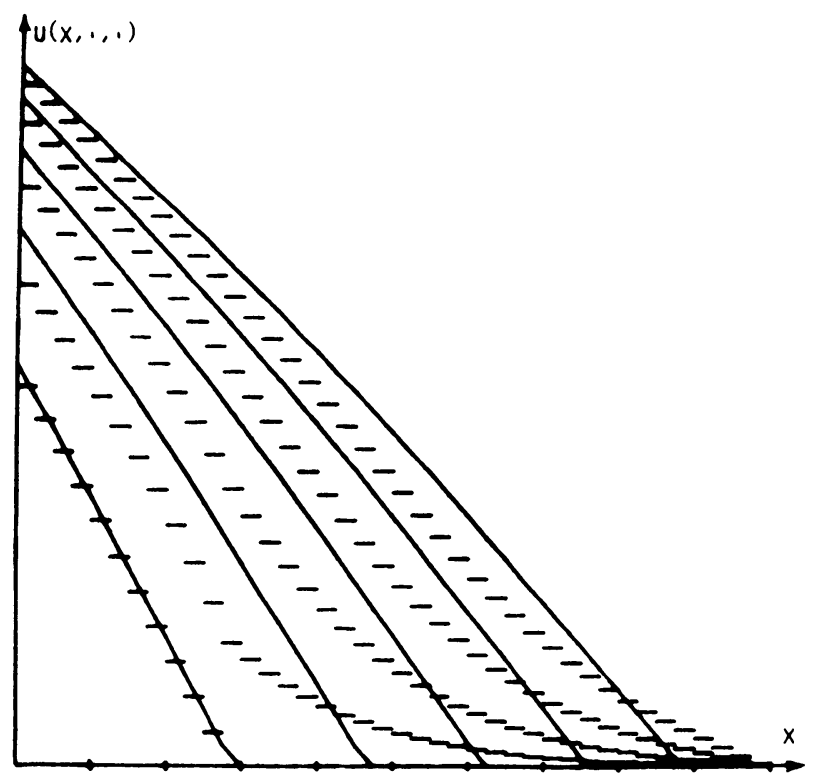

FIGURE 9

Discrete and continuous (solid lines) density for $t=0$., 0.25 , $0.5,0.75,1$. and $y=$ constant. 
Instituto de Desarrollo Tecnológico para la Industria Química

Programa Especial de Matemática Aplicada

Sante Fe, Argentina

and

Institute for Mathematics and Its Applications

Minneapolis, Minnesota

Dipartimento di Meccanica Strutturale

Università di Pavia

and

Istituto di Analisi Numerica del C.N.R.

Pavia, Italy

1. O. AXelsson \& V. A. BARKer, Finite Element Solution of Boundary Value Problems: Theory and Computation, Academic Press, Orlando, Florida, 1984.

2. A. E. BERGER, H. BREzIS \& J. C. W. ROGERS, "A numerical method for solving the problem $u_{t}-\Delta f(u)=0$," RAIRO Anal. Numér., v. 13, 1979, pp. 297-312.

3. A. Bossavit, A. Damlamian \& M. Fremond (Editors), Free Boundary Problems: Applications and Theory, Vol. III, Research Notes in Math., Pitman, Boston, 1985.

4. H. BREZIS \& A. PAZY, "Convergence and approximation of semigroups of nonlinear operators in Banach spaces," J. Funct. Anal., v. 9, 1972, pp. 63-74.

5. P. G. Ciarlet, The Finite Element Method for Elliptic Problems, North-Holland, Amsterdam, 1978.

6. P. G. CiaRlet \& P. A. RAViaRT, "Maximum principle and uniform convergence for the finite element method," Comput. Methods Appl. Mech. Engrg., v. 2, 1973, pp. 17-31.

7. J. DOUglas, JR. \& T. DUPONT, "Alternating-direction Galerkin methods on rectangles," in Numerical Solutions of Partial Differential Equations, Vol. II (B. Hubbard, ed.), Academic Press, New York, 1971, pp. 133-214.

8. C. M. ElliOTT, "Error analysis of the enthalpy method for the Stefan problem," IMA J. Numer. Anal., v. 7, 1987, pp. 61-71.

9. J. W. Jerome, Approximation of Nonlinear Evolution Systems, Academic Press, New York, 1983.

10. J. W. JEROME \& M. E. ROSE, "Error estimates for the multidimensional two-phase Stefan problem," Math. Comp., v. 39, 1982, pp. 377-414.

11. E. Magenes, "Problemi di Stefan bifase in più variabili spaziali," Le Matematiche, v. 36, 1981, pp. 65-108.

12. E. MAGENES, "Remarques sur l'approximation des problèmes non linéaires paraboliques," to appear in the volume dedicated to J. L. Lions, Gauthier-Villars, Paris, 1988.

13. E. MAgenes, R. H. Nochetto \& C. Verdi, "Energy error estimates for a linear scheme to approximate nonlinear parabolic equations," RAIRO Modél. Math. Anal. Numér., v. 21, 1987, pp. 655-678.

14. E. MAGENES \& C. VERDI, "On the semigroup approach to the two-phase Stefan problem with nonlinear flux conditions," in Free Boundary Problems: Applications and Theory, Vol. III (A. Bossavit, A. Damlamian and M. Fremond, eds.), Research Notes in Math., Pitman, Boston, 1985, pp. 28-39.

15. T. A. MANTEUfFel, "The shifted incomplete Cholesky factorization," Report Sandia Laboratories 78-8226 (1978); "An incomplete factorization technique for positive definite linear systems," Math. Comp., v. 34, 1980, pp. 473-497.

16. R. H. Nochetтo, "Error estimates for two-phase Stefan problems in several space variables, I: linear boundary conditions," Calcolo, v. 22, 1985, pp. 457-499; "II: non-linear flux conditions," Calcolo, v. 22, 1985, pp. 501-534.

17. R. H. Nochetto, "Error estimates for multidimensional Stefan problems with general boundary conditions," in Free Boundary Problems: Applications and Theory, Vol. III (A. Bossavit, A. Damlamian and M. Fremond, eds.), Research Notes in Math., Pitman, Boston, 1985, pp. 50-60.

18. R. H. Nochetto, "Error estimates for multidimensional singular parabolic problems," Japan. J. Appl. Math., v. 4, 1987, pp. 111-138.

19. R. H. NOCHETTO, "A note on the approximation of free boundaries by finite element methods," RAIRO Modél. Math. Anal. Numér., v. 20, 1986, pp. 355-368. 
20. R. H. NochetTo \& C. VeRDI, "Approximation of degenerate parabolic problems using numerical integration," SIAM J. Numer. Anal., v. 25, 1988. (To appear.)

21. M. PAOLINI, G. SACCHI \& C. VERDI, "Finite element approximations of singular parabolic problems," Internat. J. Numer. Methods Engrg. (To appear.)

22. P. A. RAVIART, "The use of numerical integration in finite element methods for solving parabolic equations," in Topics in Numerical Analysis (J. J. H. Miller, ed.), Academic Press, London, 1973, pp. 233-264.

23. M. E. ROSE, "Numerical methods for flows through porous media. I," Math. Comp., v. 40, 1983, pp. 435-467.

24. C. VERDI, "On the numerical approach to a two-phase Stefan problem with non-linear flux," Calcolo, v. 22, 1985, pp. 351-381.

25. C. VERDI, "Optimal error estimates for an approximation of degenerate parabolic problems," Numer. Funct. Anal. Optim., v. 9, 1987, pp. 657-670.

26. C. VERDI \& A. VISINTIN, "Error estimates for a semiexplicit numerical scheme for Stefantype problems," Numer. Math., v. 52, 1988, pp. 165-185.

27. A. Visintin, "Stefan problem with phase relaxation," IMA J. Appl. Math., v. 34, 1985, pp. 225-245. 